Supporting information for:

\title{
Influences on Subsurface Plutonium and Americium
}

\section{Migration}

Hilary P. Emersont, Carolyn I. Pearce*, t; Calvin H. Delegardtt; Kirk J. Cantrell; Michelle

M.V. Snydert; May-Lin Thomast, Brandy N. Gartmant; Micah D. Millert; Charles T.

Rescht; Steve M. Healds; Andrew E. Plymalet; Dallas D. Reillyt; Sarah A. Saslowt;

William Neilson $\$$; Samuel Murphy ${ }^{\$} ;$ Mavrik Zavarinł; Annie. B. Kerstingł; Vicky L.

Freedmant

†Pacific Northwest National Laboratory, Richland, WA

††TradeWind Services, LLC, Richland, WA

§Advanced Photon Source, Argonne, IL

\$ Lancaster University, Lancaster LA1 4YR, UK

‡Lawrence Livermore National Laboratory, Livermore, CA 


\section{Corresponding Author:}

*carolyn.pearce@pnnl.gov

For submission to: ACS Earth and Space Chemistry

KEYWORDS: plutonium, americium, Hanford site, fate and transport, X-ray absorption

spectroscopy; Autoradiography

Summary of Supporting Information: 41 pages (including cover page), 14 Figures, 15 Tables

\subsection{MATERIALS AND METHODS}

Table S1. Major component concentrations in the 200W Area groundwater used in these studies (adapted from [1]).

\begin{tabular}{cccc}
\hline Component & & $\begin{array}{c}\text { Initial Composition } \\
\text { Concentration (mg/L) }\end{array}$ & \\
\hline Calcium & Chloride & 59.8 & 19.1 \\
Magnesium & & 20.6 & \\
Potassium & & 7.28 & 24.2 \\
Silicon & Sodium & 28.2 & \\
& & 0.22 & \\
Strontium & & 73.7 & \\
Sulfate & & & \\
\hline
\end{tabular}

\subsection{Aqueous phase analyses}

Inductively coupled plasma-mass spectrometry (ICP-MS) 
Metals were analyzed using two different ICP-MS instruments based on equipment availability. The first instrument was an X-Series II ICP Mass Spectrometer from Thermo Fisher Scientific and the second instrument was an ELAN DRC II ICP Mass Spectrometer from PerkinElmer. High purity calibration standards were used to generate calibration curves and a separate standard source to verify continuing calibration during the analytical run. An internal standard is added to both samples and standards to correct for instrument drift and matrix effects. Dilutions of samples and standards were made in a $2 \%$ nitric acid solution, and serial dilutions were made to investigate and correct for matrix interferences. This method is based on EPA SW-846 6020A, Inductively Coupled Plasma-Mass Spectrometry [2, 3].

Inductively coupled plasma-optical emission spectrometry (ICP-OES)

Metals were analyzed quantitatively using a PerkinElmer Optima 8300 dual view ICP-

OES and a PerkinEImer S-10 auto-sampler interface. The instrument was calibrated 
using standards made by the High-Purity Standards (Charleston, SC) to generate calibration curves. All samples and standards were diluted with $2 \%$ nitric acid (Fisher

Scientific, Optima trace metal grade) and twice deionized water with resistivity no lower than $18.0 \mathrm{M} \Omega-\mathrm{cm}$. Serial dilutions were made to investigate and correct for matrix interferences. This method is similar to EPA SW-846 Method 6010C, Inductively Coupled Plasma-Atomic Emission Spectrometry [4, 5].

\section{Alkalinity}

Alkalinity was measured through titration using a Metrohm 888 Titrando Titrator controlled by a personal computer running Tiamo 2.4 software, with $0.02 \mathrm{~N}$ sulfuric acid $\left(\mathrm{H}_{2} \mathrm{SO}_{4}\right)$ to a phenolphthalein endpoint $(\mathrm{pH} 4.5)$ for total alkalinity. The alkalinity procedure is similar to Standard Methods for the Examination of Water and Wastewater, Titration Method 2320B $[6,7]$.

Anion analysis by ion chromatography (IC)

Anions were analyzed using either a Dionex Reagent Free Ion Chromatography System 5000 (RFICS-5000) with an AS-AP auto-sampler or a Dionex Reagent Free Ion 
Chromatography System 2000 (RFICS-2000) with an AS-1 auto-sampler. Both instruments were calibrated using a multi-component anion solution made by Inorganic Ventures (Christianburg, VA). Fluoride, chloride, nitrite, bromide, nitrate, phosphate and sulfate were separated on a Dionex AS17C column with a gradient elution of $1 \mathrm{mM}$ to 35 $\mathrm{mM}$ potassium hydroxide and measured using a conductivity detector. This method is similar to EPA Method 300, with the exception of using the gradient elution of potassium hydroxide $[8,9]$.

\subsection{Solid phase sample preparation}

\section{Silicon wafer preparation}

Dried sediments were resuspended in deionized water then deposited onto a silicon water substrate and allowed to dry. The samples were sealed in Kapton pockets with Mylar windows prior to removal from the contaminated area for analysis.

Thin section preparation 
Dried sediments were prepared in a $1.6 \mathrm{~cm}$ (ID) aluminum tubing with slow drying epoxy.

Two parts of epoxy were mixed with one part of hardener (EpoThin2 epoxy and hardener,

Buehler, Lake Bluff, IL) with a volume of $3 \mathrm{~mL}$ with $0.1-0.5$ grams of sediment. Samples

were then vacuum-degassed to remove bubbles and air-dried overnight at room

temperature. Slices were cut with an Isomet 1000 diamond blade thin sectioning saw with

Isocut fluid (Buehler, Lake Bluff, IL) to a thickness of approximately $150 \mu \mathrm{m}$. After

mounting on a glass microscope slide, thin sections were sanded with silicon carbide sandpaper with Isocut fluid followed by 400 grit sandpaper, then 600 grit sandpaper, and

finally 1200 grit sandpaper (Ted Pella, Inc.), all using a figure eight pattern. Final polishing was conducted with a Nylon polishing cloth and 1- $\mu \mathrm{m}$ diamond polish (Metadi II, Buehler).

Samples were washed with methanol and dried for analysis.

\subsection{Plate Autoradiography}

Silicon wafers were imaged by traditional phosphor plate autoradiography. The silicon wafer samples were positioned in an autoradiography cassette against erased GE BAS-

IP SR imaging plates and placed in a lead cave for 74 and 339 hours, depending on the 
activity of the samples. Samples were measured with and without a paper insert to block alpha particles. The exposed plates were scanned using a GE TyphoonTM FLA 9500 autoradiography scanner. The resulting imagery was normalized by the exposure time to produce maps of radioactivity as counts per hour. The exposure was conducted with and without a paper filter to differentiate the alpha emitters from other radioactive sources that are potentially present in the sediments. 
2.0 Results and discussion

\subsection{Autoradiography}
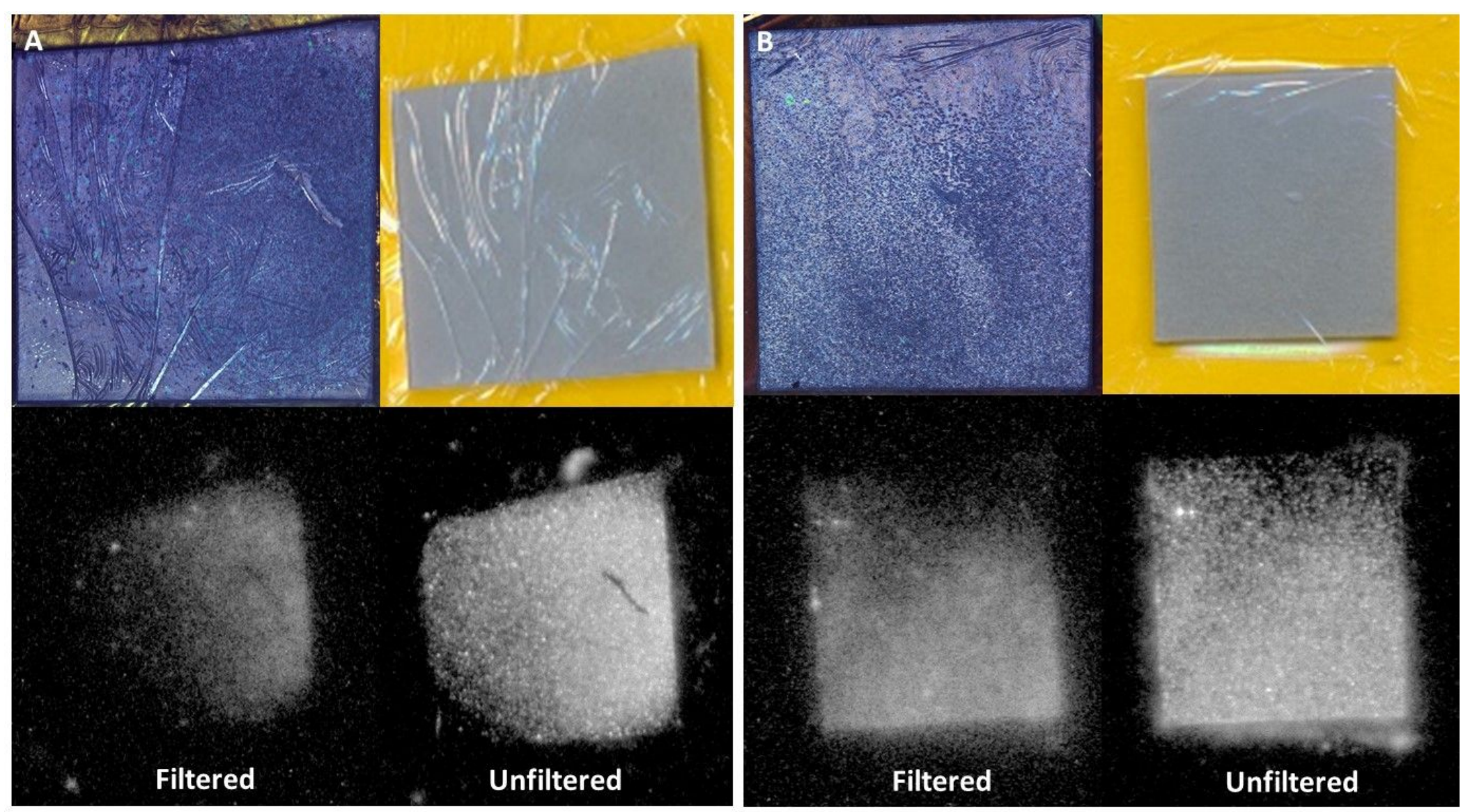
Figure S1. Autoradiography of B1HK27 (A) B1HK42 (B) samples mounted on Si wafers with and without a paper filter to selectively remove signal intensity from a-particle emission. The top right images in Figure S1 A and B show the subtraction of the filtered and unfiltered exposures overlaid on images of the sediment particles taken using a light microscopy, and the lighter spots, indicating alpha activity, were used to guide XMP mapping.

\subsection{SEM-EDX analysis}




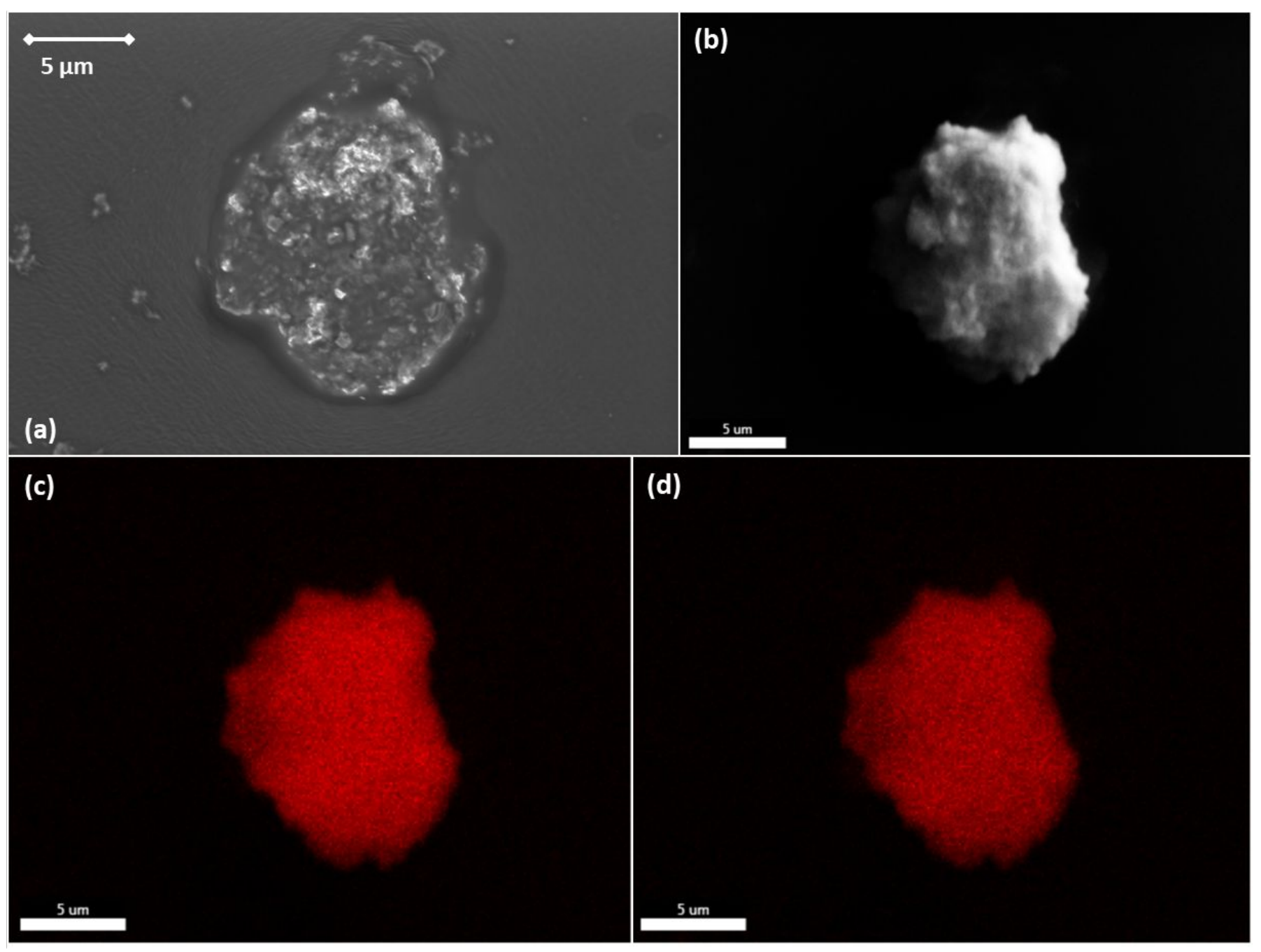

Figure S2. Electron micrographs collected with the SEM backscatter detector representing Pu/Am phase observed in sediment sample Z9-4-11A following preparation of dried sediment on silicon wafers with approximately $20 \mathrm{~nm}$ carbon coating for (a) $5 \mathrm{keV}$ operating voltage and (b) $25 \mathrm{keV}$ operating voltage showing differences in depth penetration with 
voltage with EDX mapping for $\mathrm{Am}$ (c) and $\mathrm{Pu}$ (d). Note: additional trace elements were also associated with particles including but not limited to $\mathrm{Ni}, \mathrm{Nd}, \mathrm{Sb}, \mathrm{Cu}$, and $\mathrm{Re}$.

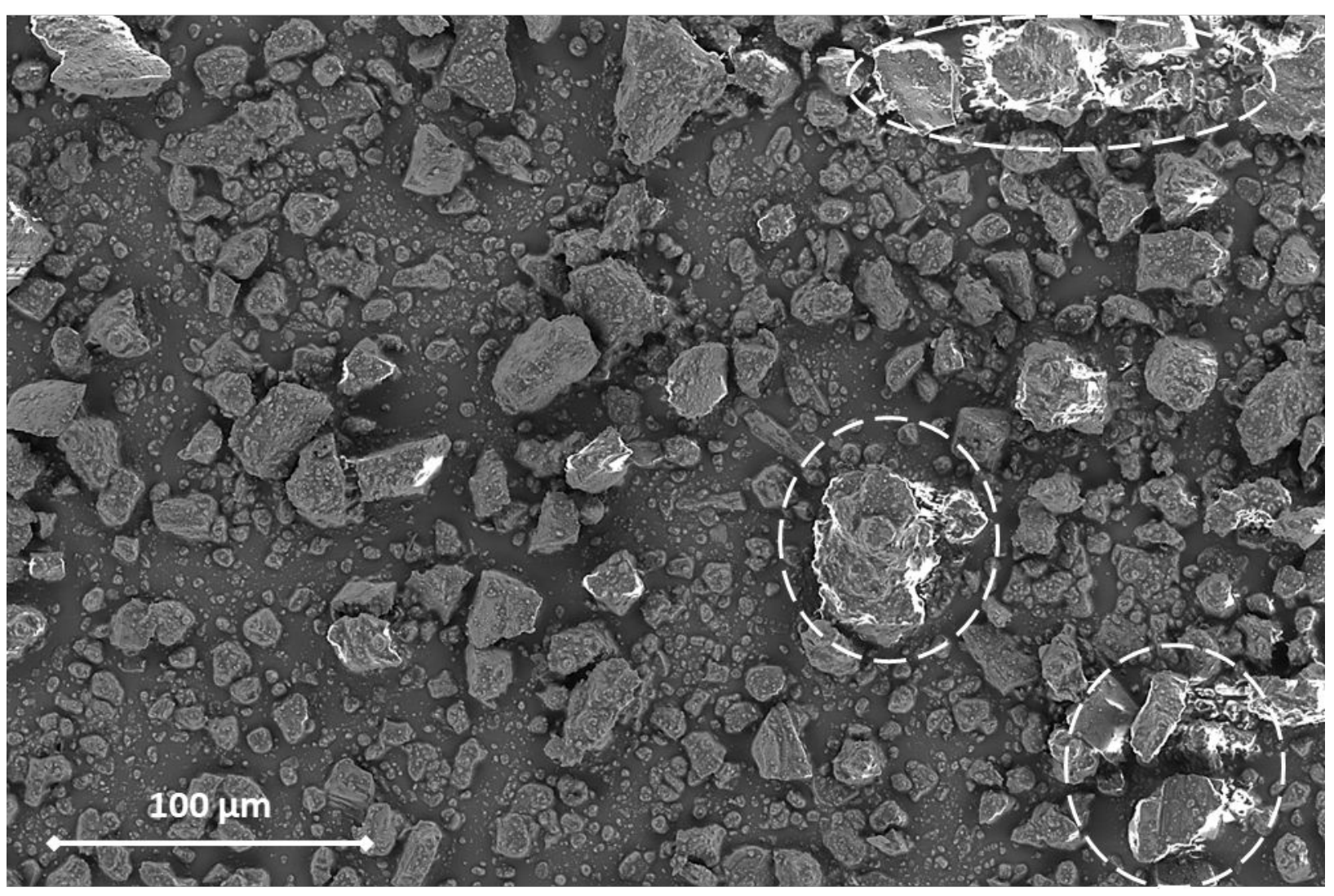

Figure S3. SEM images of Z-12 sediments collected with the backscatter detector. Pu and Am not observed via EDS in any of the 10 regions observed likely because $\mathrm{Pu}$ is spread more homogenously as compared to $\mathrm{Z9}$ sediments as either adsorbed or small $\mathrm{PuO}_{2}$ precipitates, although significant charging of these samples was observed as shown in the white dashed lines. 


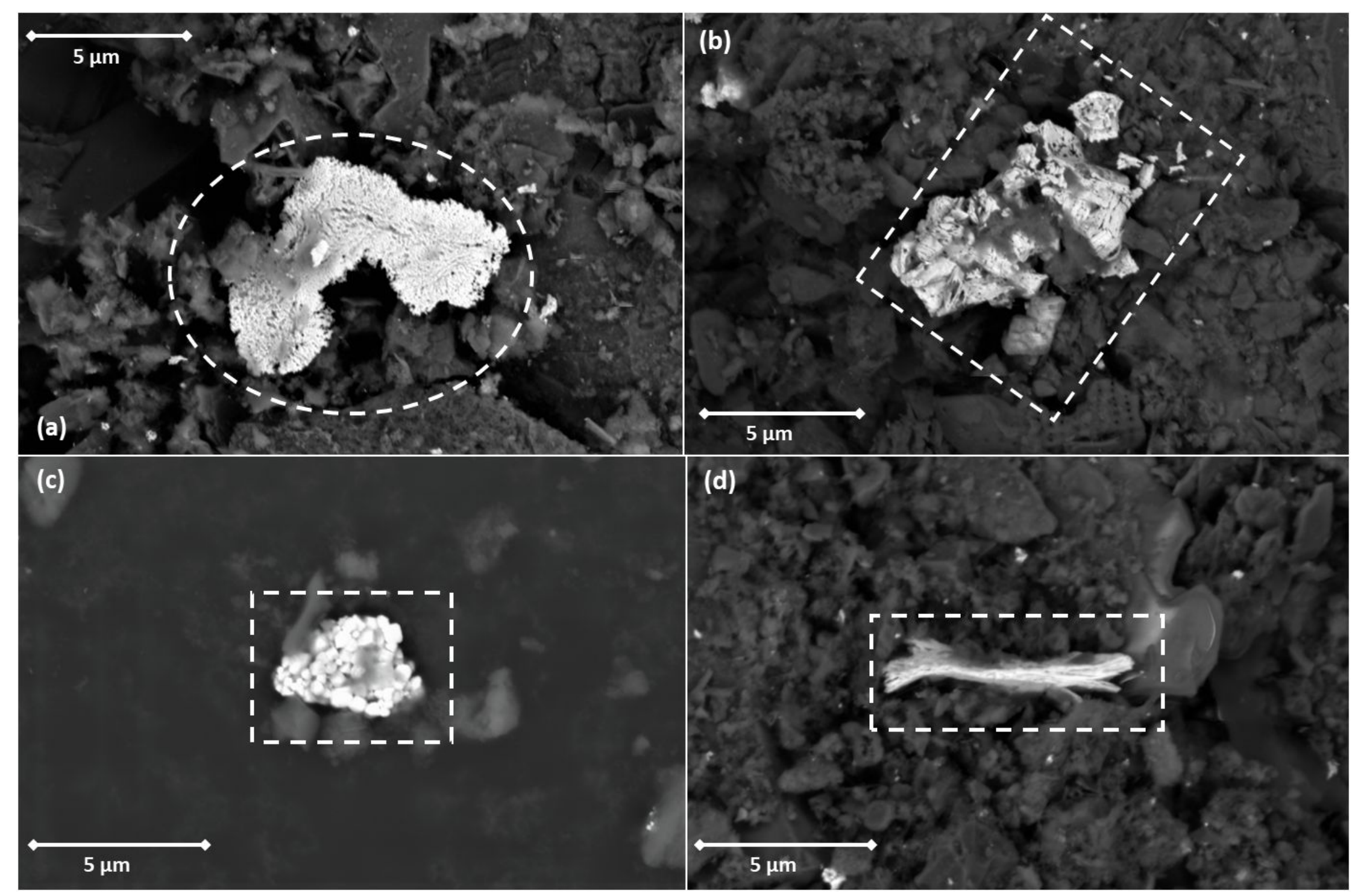


Figure S4. Pu aggregate morphology as shown by SEM backscatter detector images following $8 \mathrm{M} \mathrm{HNO}_{3}$ leaching in Z9-4$5 A$ sediments for (a) and (b) and Z9-4-11A sediments for (c) and (d).

\section{$2.3 X$-ray microprobe mapping}
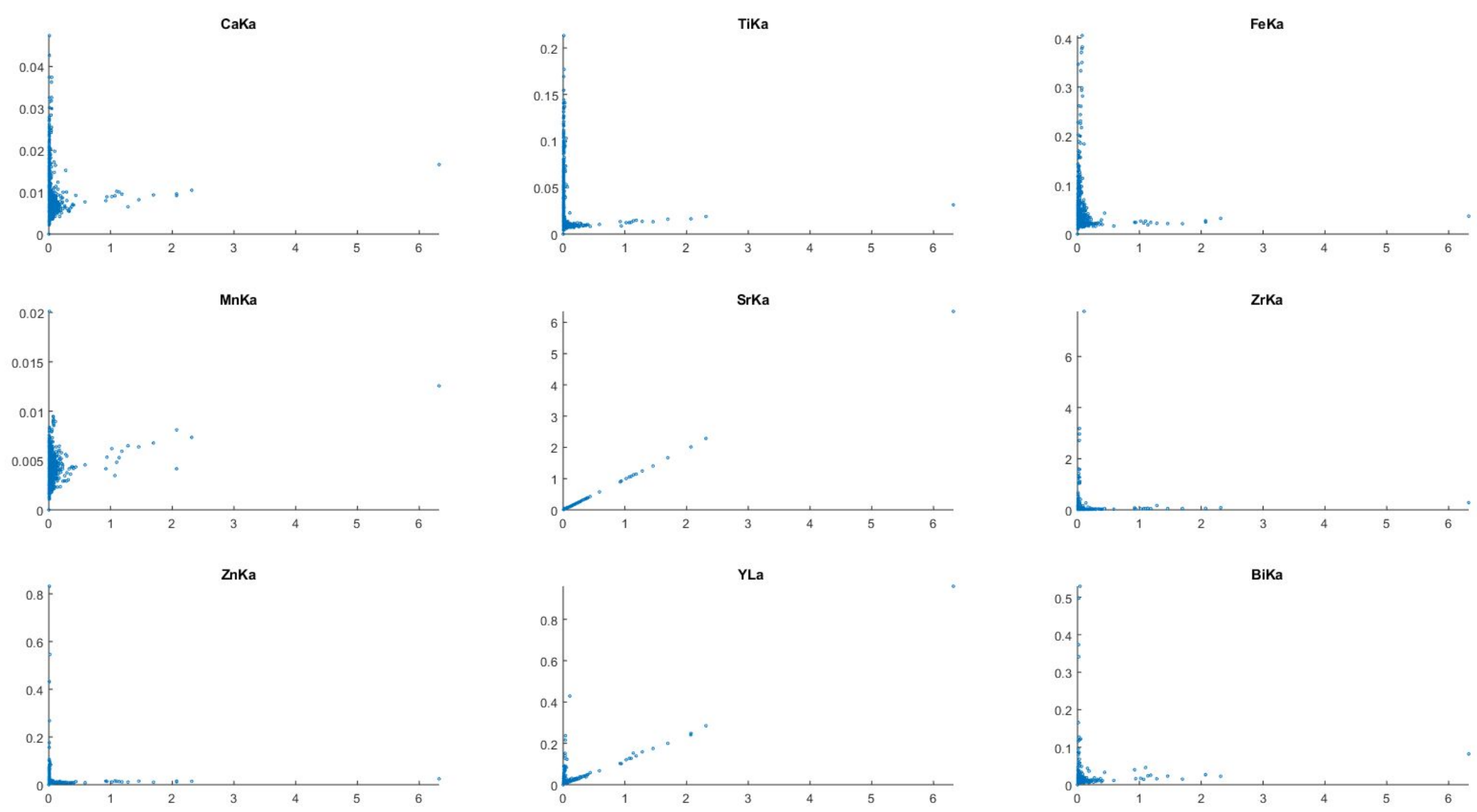
Figure S5. Correlation of elements with the plutonium L-alpha fluorescence line (14282 eV) based on 2-D mapping of location \#002 of Z9-4-5A based on $\mu$-XRF analysis collected at the 20-ID-B,C beamline at the Advanced Photon Source at Argonne National Laboratory with the $x$-axis representing the counts measured for Pu and the $y$-axis representing the counts measured for the element listed above each figure inset. 

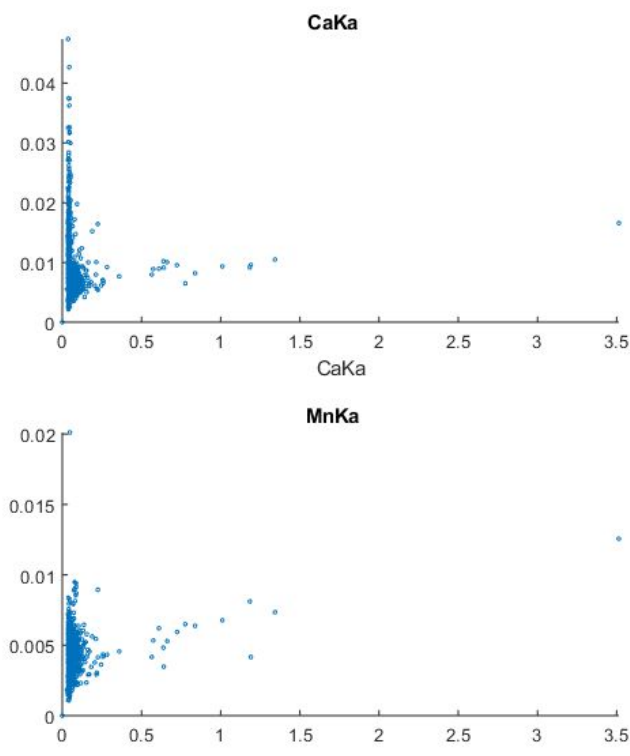

ZnKa

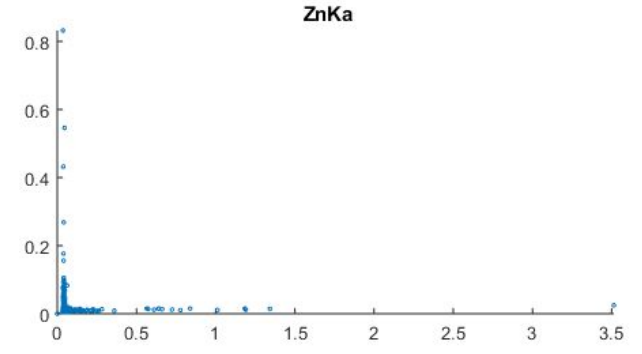

TiKa

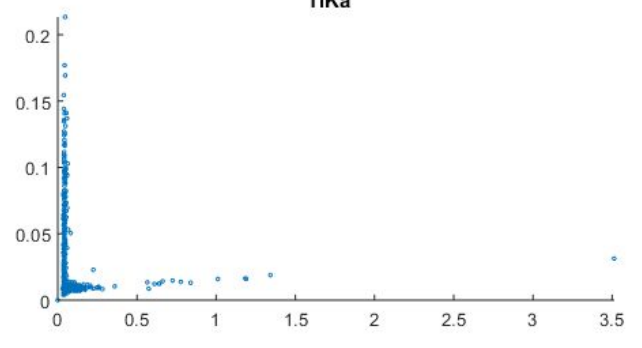

SrKa

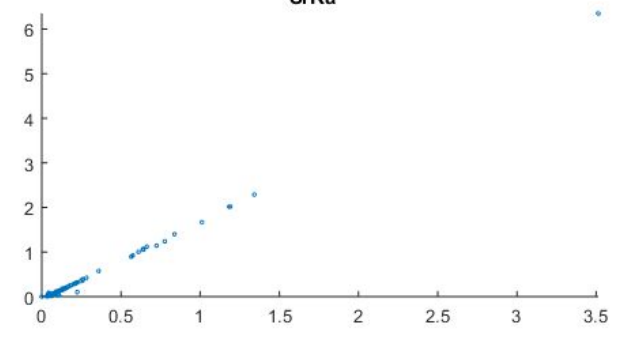

YLa

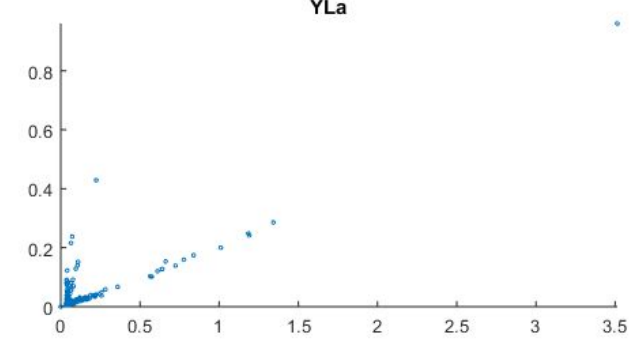

FeKa

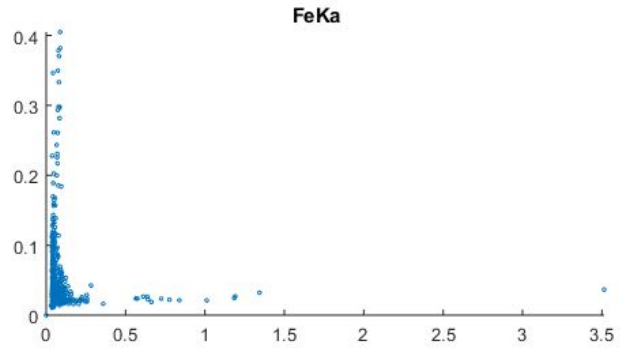

ZrKa

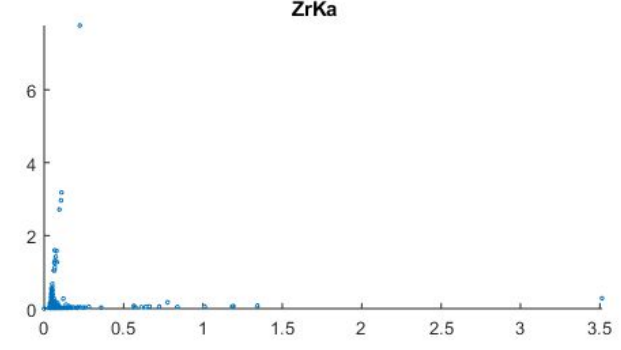

BiKa

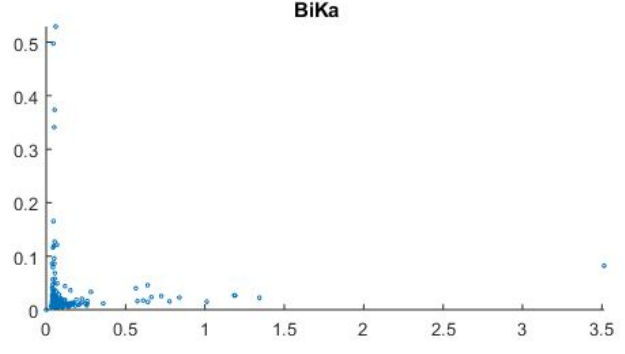

Figure S6. Correlation of elements with the plutonium L-beta fluorescence line (18296 eV) based on 2-D mapping of location \#002 of Z9-4-5A based on $\mu$-XRF analysis collected at the 20-ID-B,C beamline at the Advanced Photon Source at Argonne 
National Laboratory with the $\mathrm{x}$-axis representing the counts measured for $\mathrm{Pu}$ and the $\mathrm{y}$-axis representing the counts measured for the element listed above each figure inset. 


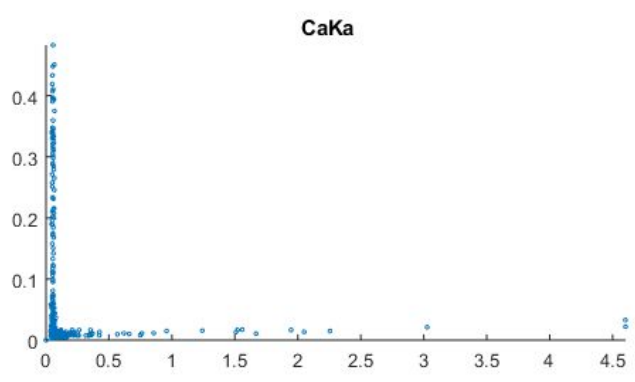

MnKa

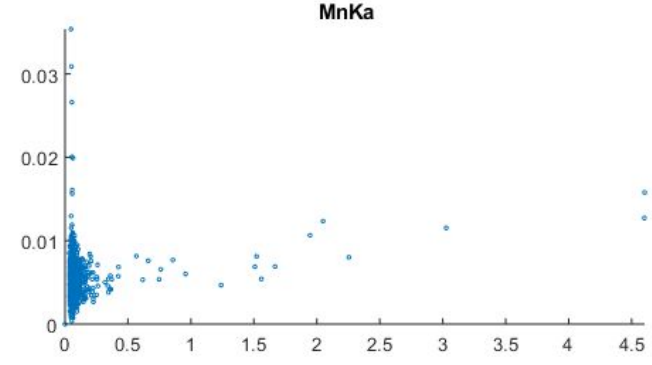

ZnKa

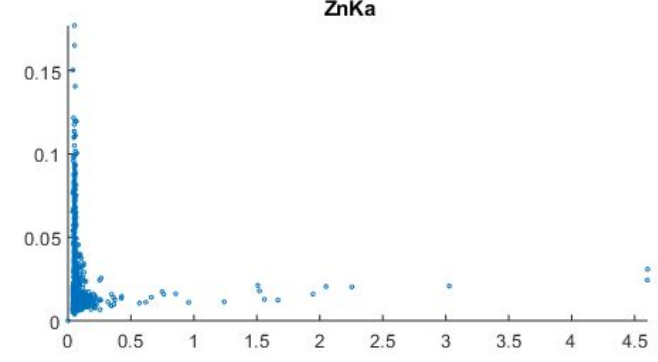

TiKa

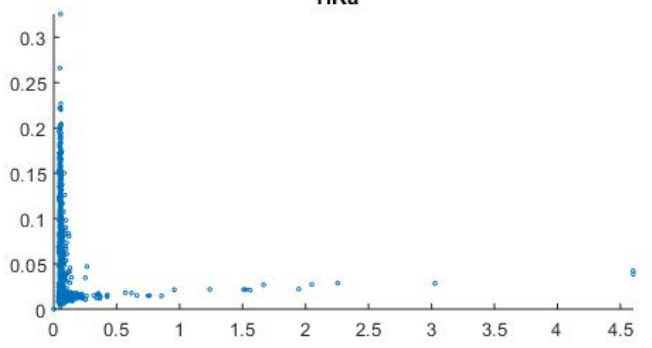

SrKa

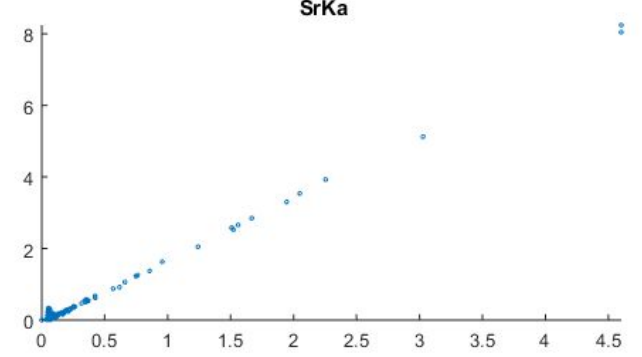

YKa

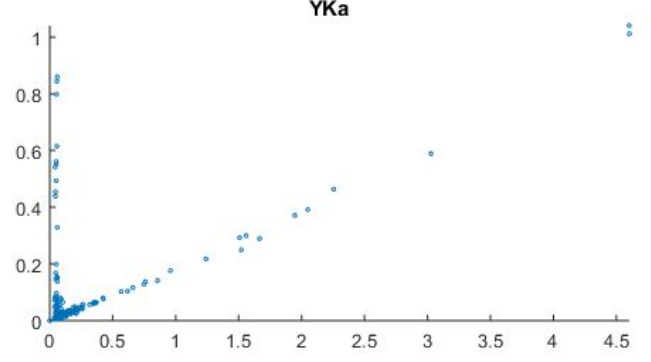

FeKa

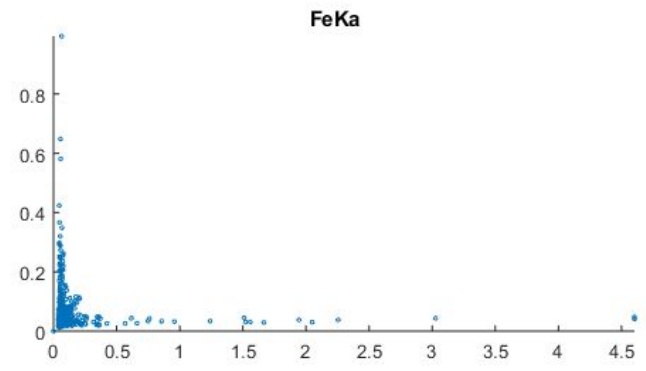

ZrKa

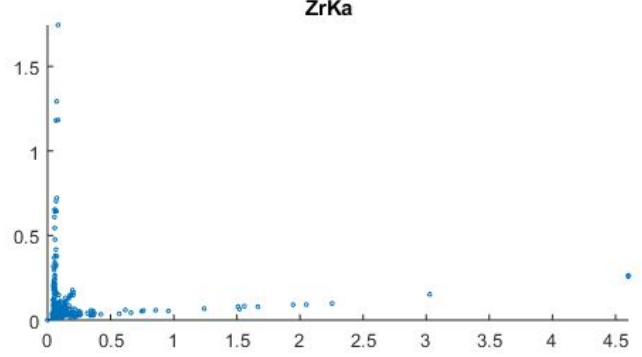

BiLa

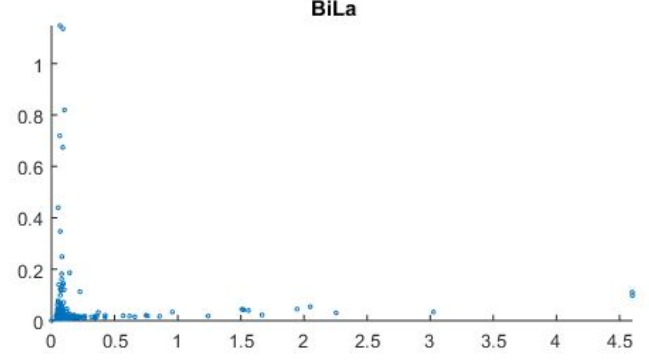

Figure S7. Correlation of elements with the plutonium L-beta fluorescence line (18296 eV) based on 2-D mapping of location \#003 of Z9-4-5A based on $\mu$-XRF analysis collected at the 20-ID-B,C beamline at the Advanced Photon Source at Argonne 
National Laboratory with the $\mathrm{x}$-axis representing the counts measured for $\mathrm{Pu}$ and the $\mathrm{y}$-axis representing the counts measured for the element listed above each figure inset.

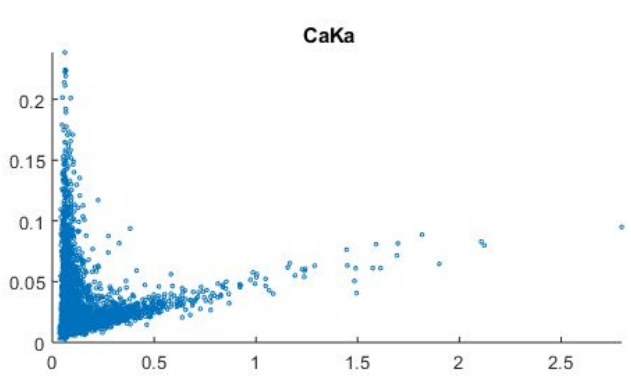

MnKa

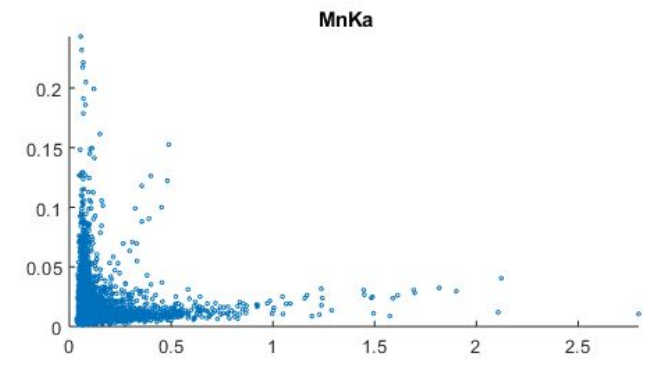

ZnKa

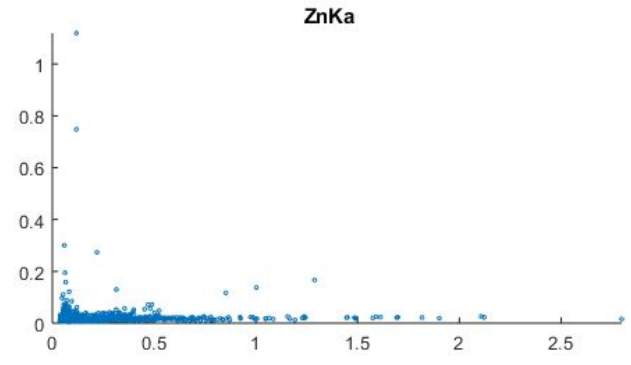

TiKa
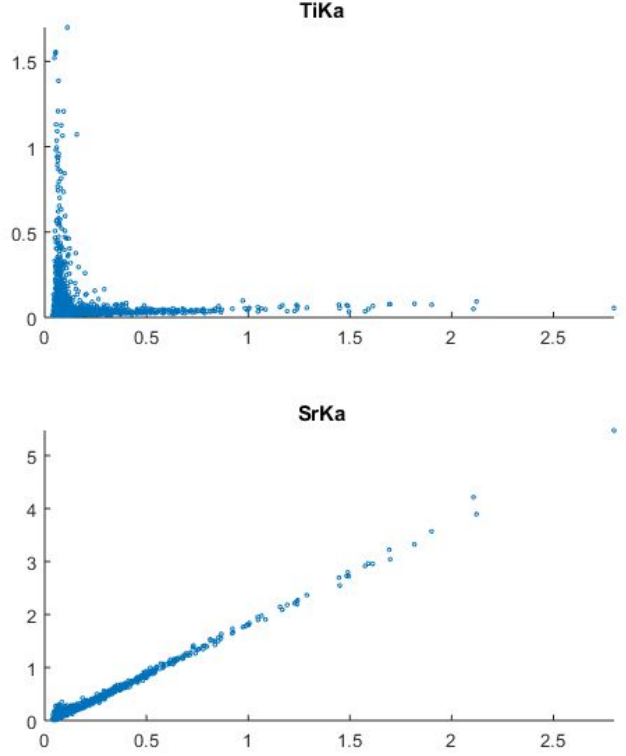

YKa

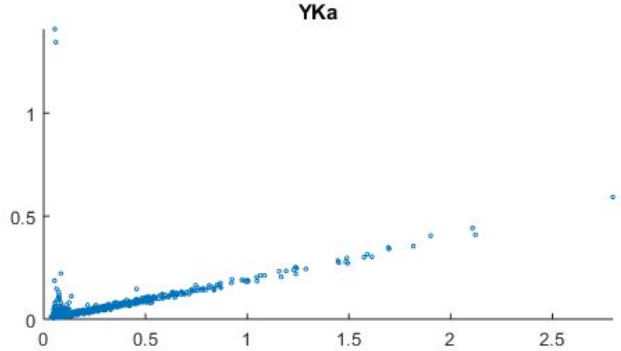

FeKa

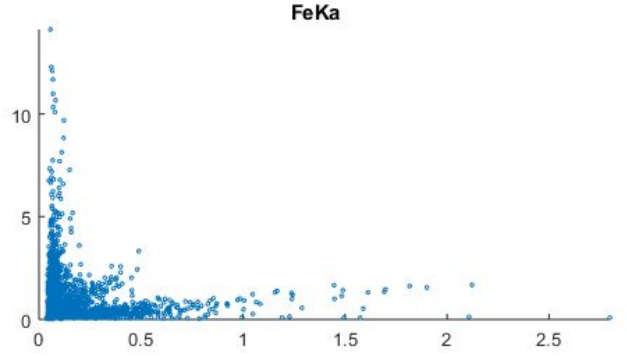

ZrKa

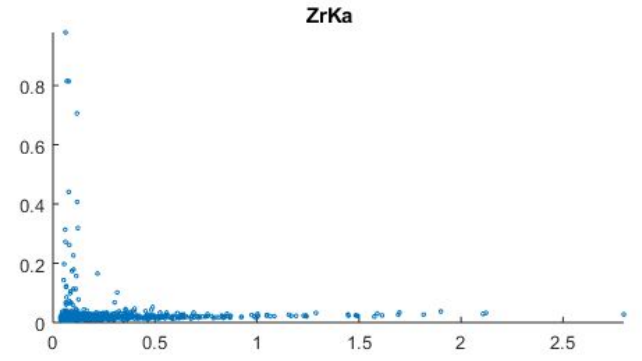

BiLa

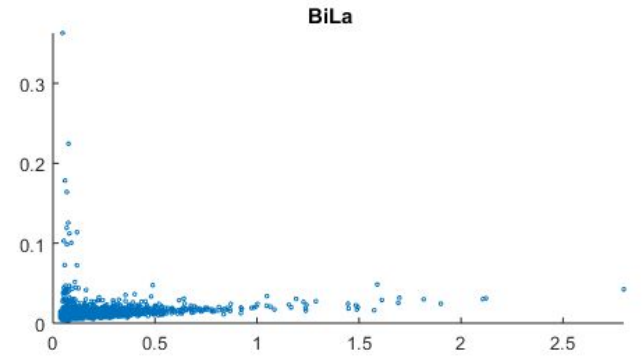


Figure S8. Correlation of elements with the plutonium L-beta fluorescence line (18296 eV) based on 2-D mapping of location \#001 of Z9-4-11A based on $\mu$-XRF analysis collected at the 20-ID-B,C beamline at the Advanced Photon Source at Argonne National Laboratory with the $\mathrm{x}$-axis representing the counts measured for $\mathrm{Pu}$ and the $\mathrm{y}$-axis representing the counts measured for the element listed above each figure inset. 


\section{$2.4 X$-ray microprobe elemental analysis}

Two-dimensional X-ray microprobe mapping was used to probe elements for which $\mathrm{Pu}$ may be associated in the solid phase. These results suggest some correlation of Pu with $\mathrm{Ca}, \mathrm{Mn}, \mathrm{Fe}, \mathrm{Ti}$, and $\mathrm{Zn}$, although many Pu particles are not strongly associated with other elements as shown by mapping figures, which suggests the existence of discrete $\mathrm{Pu}$ particles. For example, Z9-4-5A maps in SI Figures S6-S9 show some correlation with $\mathrm{Ca}$ and $\mathrm{Mn}$. Some Pu-rich inclusions have been noted in $\mathrm{CaO} / \mathrm{CaF}_{2}$ particles in scrap solids less than $90 \mu \mathrm{m}$ in size [10]. In the location mapped for Z9-4-5A in Figure S8 in SI, $\mathrm{Pu}$ is associated with lower concentrations of $\mathrm{Ti}$ and $\mathrm{Zn}$ with some correlation of $\mathrm{Mn}$ and $\mathrm{Pu}$ (signal recorded by fluorescence detector is directly correlated with concentration, although results are qualitative due to a lack of standard). A strong correlation is also observed for $\mathrm{Sr}$ and $\mathrm{Y}$, although this is due to peak overlap with $\mathrm{U}$. When graphed as a 2-D figure with the signals overlaid, there is a slight correlation with $\mathrm{Mn}$ in Figure $\mathrm{S7}$, as well as a weak association between $\mathrm{Fe}$ and $\mathrm{Ca}$ in Figure S8. 
Despite the presence of alpha emitters in the slant borehole samples, as confirmed by autoradiography, Pu was not present in high enough concentrations to be mapped by XMP in these samples. Kersting et al. (unpublished) have used NanoSIMS analysis to show that $\mathrm{Pu}$ is associated, at very low concentrations, with the mineral phases present in Hanford sediments and not isolated as a primary $\mathrm{PuO}_{2}$ phase (intrinsic Pu oxides). 

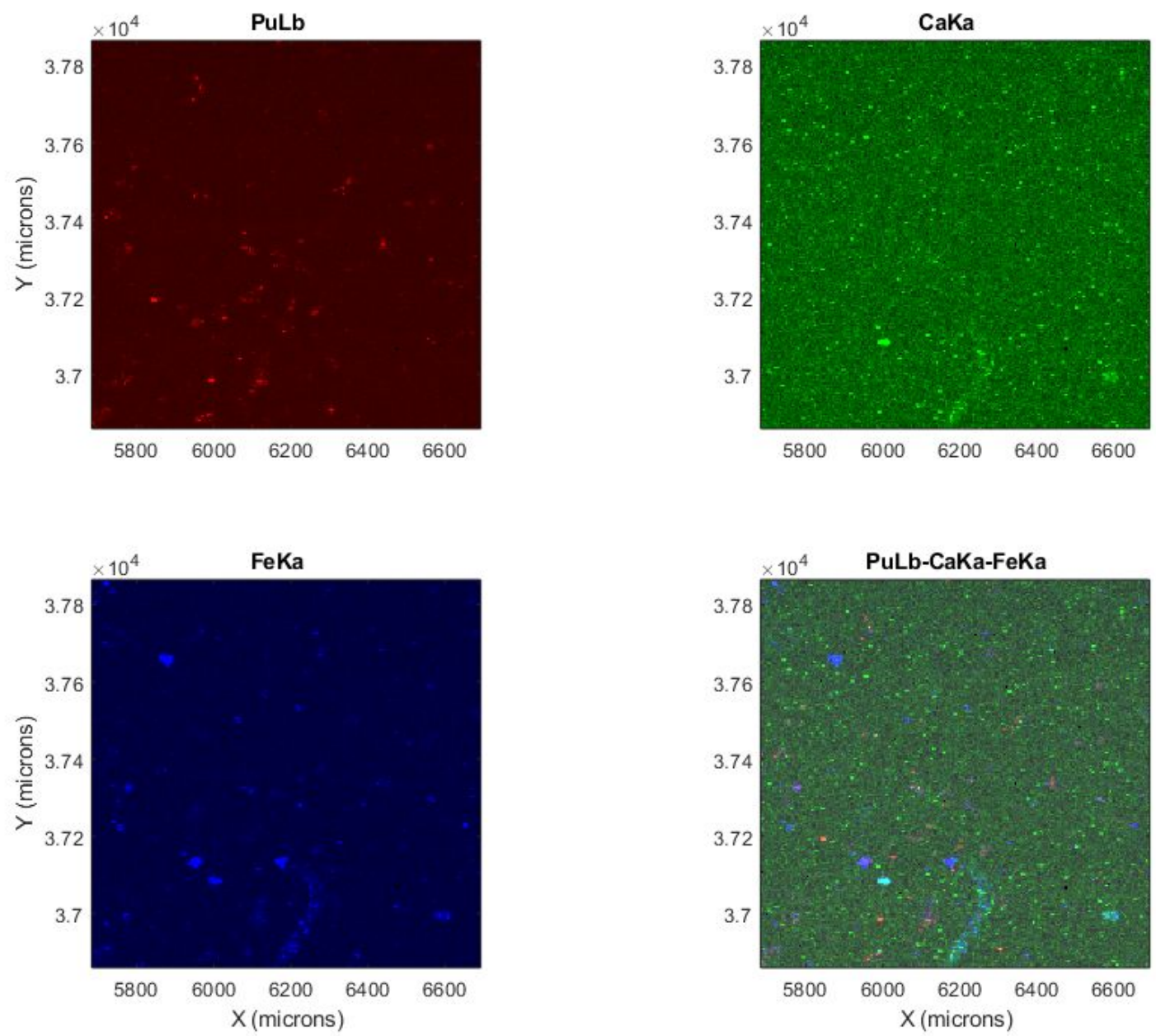

Figure S9. Correlation of iron and calcium K-alphas with the plutonium L-beta fluorescence line (18296 eV) based on 2-D mapping of location \#002 of Z9-4-5A at the 20-ID-B, C beamline at the Advanced Photon Source at Argonne National Laboratory. 

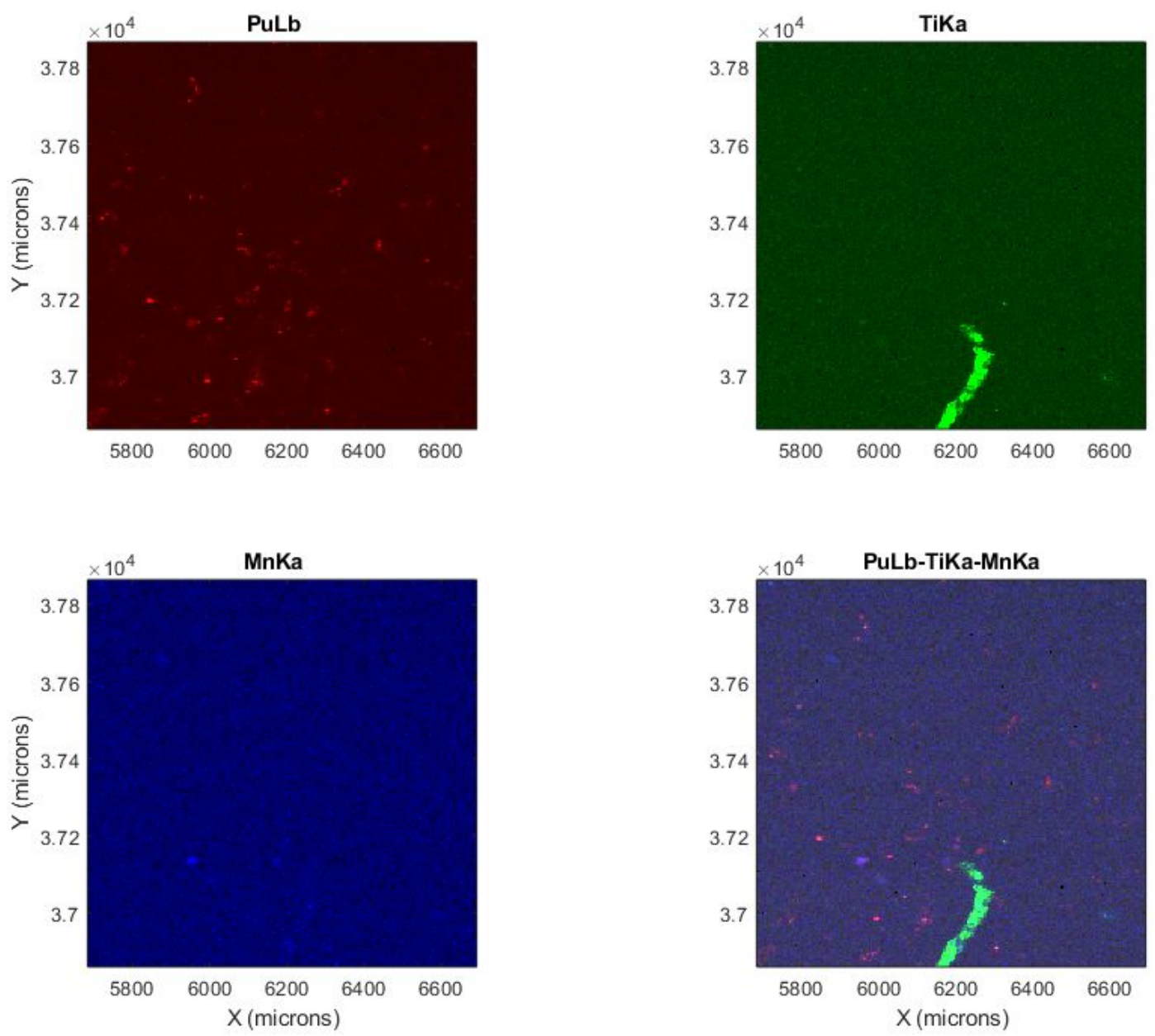

Figure S10. Correlation of titanium and manganese K-alphas with the plutonium L-beta fluorescence line (18296 eV) based on 2-D mapping of location \#002 of Z9-4-5A at the 20-ID-B, C beamline at the Advanced Photon Source at Argonne National Laboratory. 

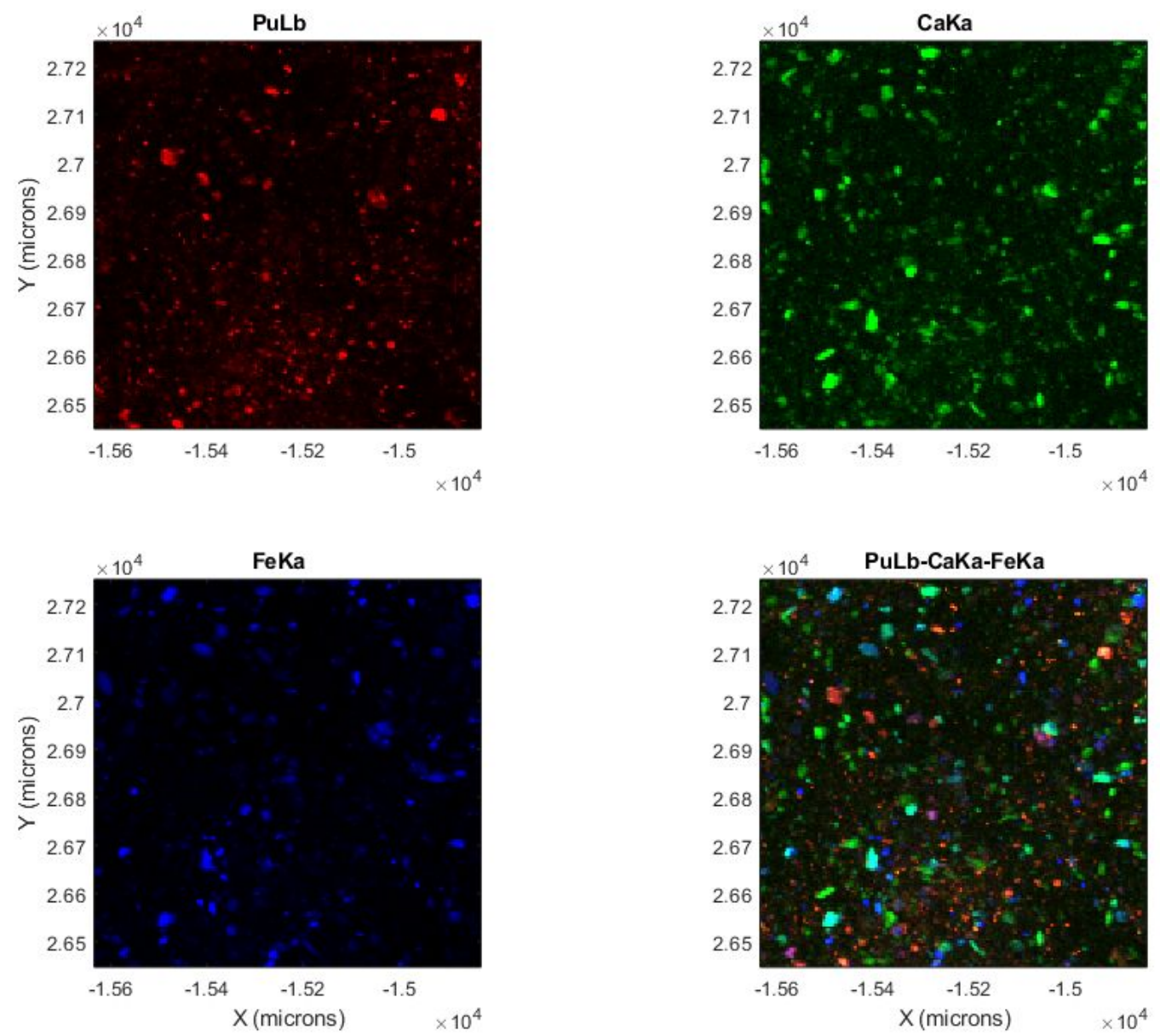

Figure S11. Correlation of iron and calcium K-alphas with the plutonium L-beta fluorescence line (18296 eV) based on 2-D mapping of location \#001 of Z9-4-11A at the 20-ID-B, C beamline at the Advanced Photon Source at Argonne National Laboratory. 


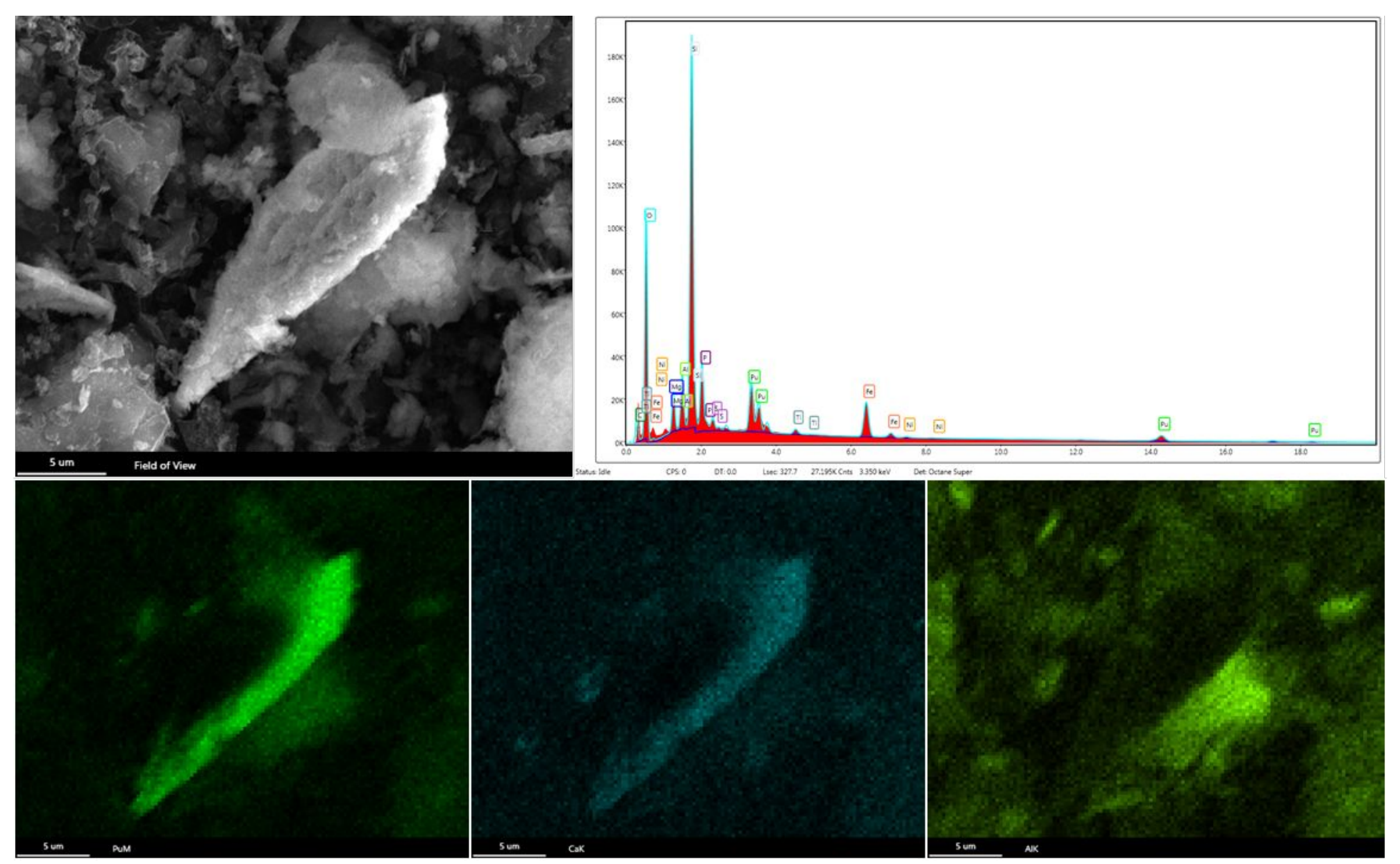

Figure S12. Pu aggregate associated with sediments from sample Z9-4-5A with trace metals adsorbed to surface (e.g., Ti, not shown) and significant $\mathrm{Ca}$ association with Al particle nearby. Note: Preparation of dried sediment on silicon wafers with approximately $20 \mathrm{~nm}$ carbon coating. 


\subsection{Comparison of Rai et al. 1980 with previously unpublished data from Delegard}

Two parallel saturated column leaching tests of the same Z-1A sediment were conducted previously to determine the extent of $\mathrm{Am}$ and $\mathrm{Pu}$ mobilization under groundwater intrusion or massive surface flooding conditions [11]. The surface flood was simulated using $0.01 \mathrm{M} \mathrm{CaCl}_{2}$ solution (30 mM ionic strength; $\mathrm{pH} 6.2$ ) and the synthetic Hanford-affected groundwater was $\sim 1.0 \mathrm{mM} \mathrm{Na}+0.17 \mathrm{mM} \mathrm{K}^{+}, 0.5 \mathrm{mM} \mathrm{Mg}^{2+}, 0.5 \mathrm{mM}$ $\mathrm{Ca}^{2+}, 1.1 \mathrm{mM} \mathrm{H}_{4} \mathrm{SiO}_{4}, 0.7 \mathrm{mM} \mathrm{NO}_{3}^{-}, 0.4 \mathrm{mM} \mathrm{SO}_{4}{ }^{2-}$, and $1.3 \mathrm{mM} \mathrm{HCO}_{3}^{-}$(4.5 mM ionic strength; $\mathrm{pH} 8.1$ ). Solutions were introduced to the bottom of $2.5-\mathrm{cm}$ diameter and $22-\mathrm{cm}$ long packed (180-grams of sediment) in glass columns with leach solutions pumped upward to ensure saturation. The solutions were added at a $\sim 2.6 \mathrm{~cm} /$ hour flow rate through the sediment. Effluent solutions were collected and analyzed for $\mathrm{pH}, \mathrm{Am}, \mathrm{Pu}$, and chemical solute concentrations. The Pu and Am initially eluted in a similar pattern but the Pu activities in solution were about two orders of magnitude lower than those of Am on a molar basis, which meant that the Pu concentrations soon went below alpha spectrometry detection. The Am elution concentrations as functions of $\mathrm{pH}$ are compared with the concentrations observed by Rai et al. (1981) [12]. 
The Am concentrations in the first two pore volumes exceeded the concentrations expected based on batch tests. Over $80 \%$ of the Am ultimately leached was removed in the first 210 of the pore volumes. The $\mathrm{pH}$ generally increased with added solution, but the waste-affected sediment was buffered well below that normally found in native Hanford sediments $(\mathrm{pH} \sim 8)$. After 210 pore volumes, only $\sim 30 \%$ of the Am and less than $1 \%$ of the $\mathrm{Pu}$ were removed from the sediment by each solution. The chloride, fluoride, and nitrate elution were slightly retarded in the column tests indicating that the sediment behaved as a weak anion exchanger. The anion retardation behavior likely was due to the low waste $\mathrm{pH}$ which protonated the sediment minerals to impart a net positive charge. This effect could have been magnified by hydrated iron oxide, precipitated from dissolved iron in the waste, to give more exchange sites. 


\subsection{Extraction of other elements in batch sediment extractions}

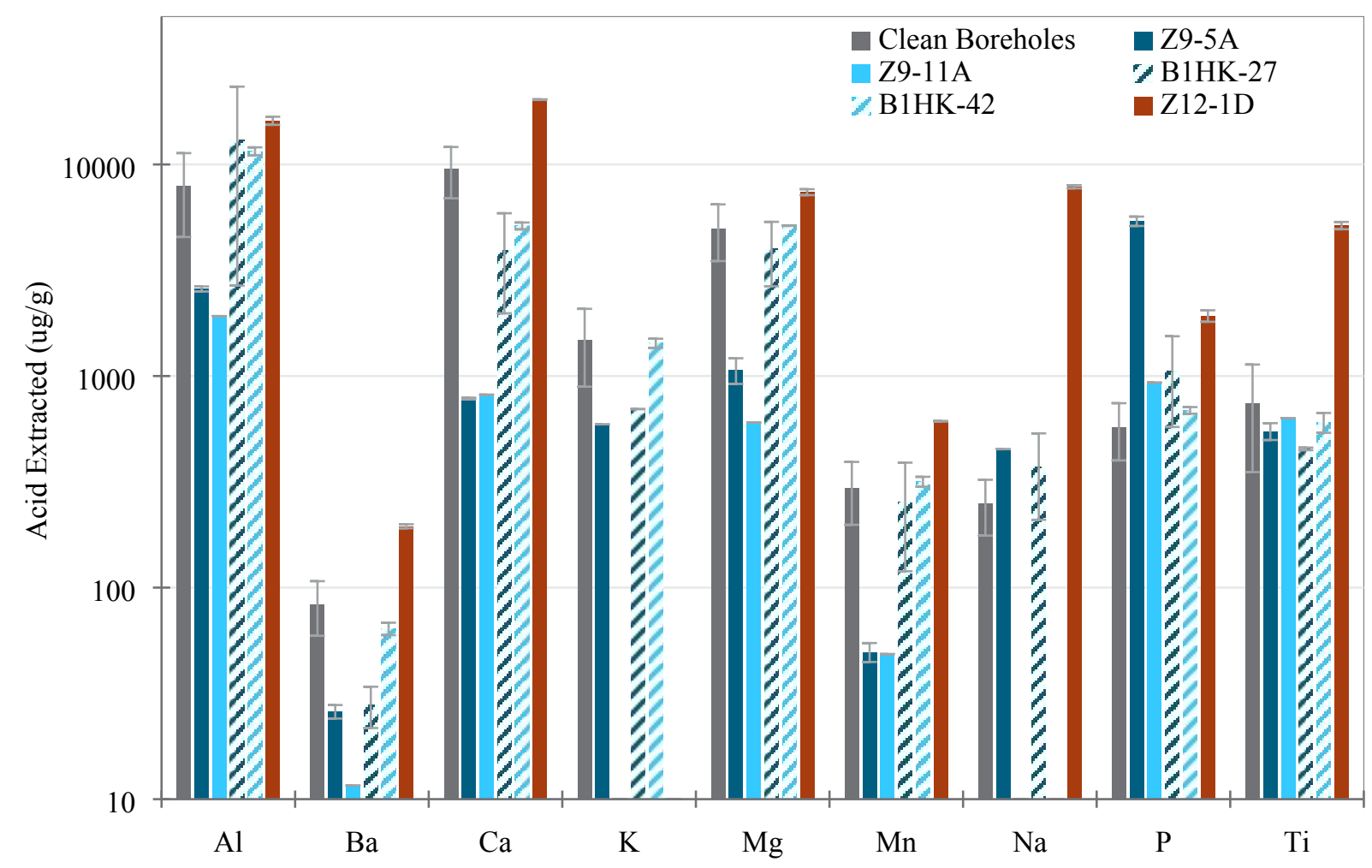

Figure S13. Comparison of major elements $(\mu \mathrm{g} / \mathrm{g})$ measured in acid extractions with two 'clean' boreholes analyzed previously [13] and crib sediments (this research), Note: Only shown for elements which are above detection limits, standard deviation based on duplicate analysis for crib samples. 


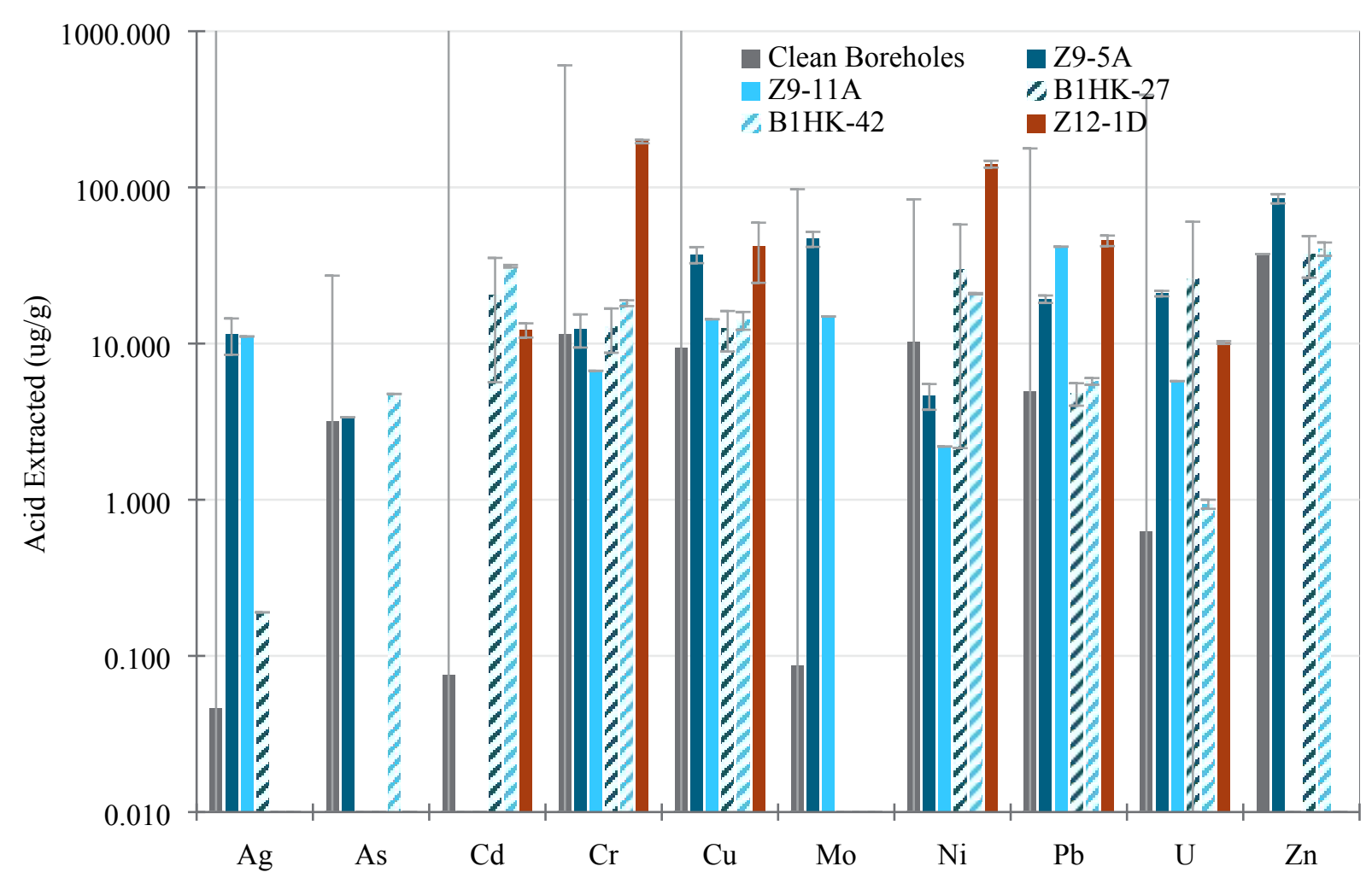

Figure S14. Comparison of trace metals $(\mu \mathrm{g} / \mathrm{g})$ measured in acid extractions with two 'clean' boreholes analyzed previously [13] and crib sediments (this research), Note: Only shown for elements which are above detection limits, standard deviation based on duplicate analysis for crib samples. 
Table S2. Z-9 and Z-12 sediment sample extraction results (by mass, in $\mu \mathrm{g} / \mathrm{g}$ ) for $\mathrm{U}$ and Th as determined by ICP-MS, relative standard deviation (\%) is based on analysis of two duplicate samples.

\begin{tabular}{|c|c|c|c|c|}
\hline \multirow[b]{2}{*}{ Sample ID } & \multicolumn{2}{|c|}{ Acid Extracts } & \multicolumn{2}{|c|}{ Groundwater Extracts } \\
\hline & $\begin{array}{l}{ }^{232} \mathrm{Th} \\
(\mu \mathrm{g} / \mathrm{g})\end{array}$ & $\begin{array}{c}{ }^{238} \mathrm{U} \\
(\mu \mathrm{g} / \mathrm{g})\end{array}$ & $\begin{array}{l}{ }^{232} \mathrm{Th} \\
(\mu \mathrm{g} / \mathrm{g})\end{array}$ & $\begin{array}{c}U^{\mathrm{a}} \\
(\mu \mathrm{g} / \mathrm{g})\end{array}$ \\
\hline Z9-4-5A & $0.870(6.3 \%)$ & $20.9(4.1 \%)$ & $0.006(17.7 \%)$ & $0.104(1.4 \%)$ \\
\hline Z9-4-11A & 0.577 (NA) & 5.75 (NA) & $0.010(0.0 \%)$ & $0.048(4.1 \%)$ \\
\hline B1HK-27 & $2.20(14.8 \%)$ & $25.9(133 \%)$ & $0.002(37.2 \%)$ & $0.002(69.9 \%)$ \\
\hline B1HK-42 & $3.72(14.8 \%)$ & $0.938(6.7 \%)$ & $0.008(68.4 \%)$ & 0.003 (NA) \\
\hline Z12-1D & $2.98(1.9 \%)$ & $10.2(2.1 \%)$ & $0.018(71.7 \%)$ & $0.073(4.6 \%)$ \\
\hline
\end{tabular}

NA - Z9-4-11A duplicate sample not analyzed, B1HK42 duplicate below detection limits 
Table S3. Z-9 and Z-12 sediment sample acid extraction results (results reported as wet weight) for major and trace elements.

\begin{tabular}{|c|c|c|c|c|c|c|c|c|c|c|}
\hline Sample ID & ${ }^{107} \mathrm{Ag}(\mu \mathrm{g} / \mathrm{g})$ & $\mathrm{Al}(\mu \mathrm{g} / \mathrm{g})$ & ${ }^{75} \mathrm{As}(\mu \mathrm{g} / \mathrm{g})$ & $B^{b}(\mu g / g)$ & ${ }^{138} \mathrm{Ba}(\mu \mathrm{g} / \mathrm{g})$ & $\mathrm{Be}(\mu \mathrm{g} / \mathrm{g})$ & $\mathrm{Bi}(\mu \mathrm{g} / \mathrm{g})$ & $\mathrm{Ca}(\mu \mathrm{g} / \mathrm{g})$ & ${ }^{111} \mathrm{Cd}(\mu \mathrm{g} / \mathrm{g})$ & Co $(\mu g / g)$ \\
\hline Z9-4-5A & 9.36 & 2,530 & 3.37 & $<537$ & 24.6 & $<4.3$ & 322 & 777 & $<0.67$ & $<27$ \\
\hline Z9-4-5A dup & 13.6 & 2,630 & $<7.23$ & $<989$ & 27.3 & $<7.9$ & 341 & 788 & $<1.2$ & $<49$ \\
\hline Z9-4-11A & 11.1 & 1,920 & $<2.3$ & $<822$ & 11.6 & $<6.6$ & $<81$ & 816 & $<1.0$ & $<41$ \\
\hline Z9-4-11A dup & NA* & NA & NA & NA & NA & NA & NA & NA & NA & NA \\
\hline B1HK-27 & $<0.73$ & 20,300 & $<1.7$ & $<628$ & 23.5 & $<5.0$ & $<62$ & 5,310 & 31.0 & $<31$ \\
\hline B1HK-27 dup & 0.19 & 5,700 & $<2.1$ & $<285$ & 32.2 & $<2.3$ & $<28$ & 2,550 & 10.0 & $<14$ \\
\hline B1HK-42 & $<1.0$ & 11,900 & 4.76 & $<862$ & 67.0 & $<6.9$ & $<85$ & 4,990 & 31.7 & $<43$ \\
\hline B1HK-42 dup & $<0.41$ & 11,200 & $<4.6$ & $<628$ & 60.9 & $<5.0$ & $<62$ & 5,260 & 30.9 & $<31$ \\
\hline Z12-1D & $<6.1$ & 16,600 & $<15$ & $<5,250$ & 198 & $<42$ & $<516$ & 20,300 & 13.1 & $<262$ \\
\hline Z12-1D dup & $<3.9$ & 15,600 & $<44$ & $<5,970$ & 192 & $<48$ & $<586$ & 20,200 & 11.3 & $<297$ \\
\hline
\end{tabular}

(continued). Z-9 and Z-12 sediment sample acid extraction results.

\begin{tabular}{|c|c|c|c|c|c|c|c|c|c|c|c|}
\hline Sample ID & ${ }^{53} \mathrm{Cr}(\mu \mathrm{g} / \mathrm{g})$ & ${ }^{133} \mathrm{Cs}(\mu \mathrm{g} / \mathrm{g})$ & ${ }^{65} \mathrm{Cu}(\mu \mathrm{g} / \mathrm{g})$ & $\mathrm{Fe}(\mu \mathrm{g} / \mathrm{g})$ & ${ }^{69} \mathrm{Ga}(\mu \mathrm{g} / \mathrm{g})$ & ${ }^{157} \mathrm{Gd}(\mu \mathrm{g} / \mathrm{g})$ & ${ }^{200} \mathrm{Hg}(\mu \mathrm{g} / \mathrm{g})$ & $\mathrm{K}(\mu \mathrm{g} / \mathrm{g})$ & $\mathrm{Li}^{\mathrm{a}}(\mu \mathrm{g} / \mathrm{g})$ & $\mathrm{Mg}(\mu \mathrm{g} / \mathrm{g})$ & Mn $(\mu \mathrm{g} / \mathrm{g})$ \\
\hline Z9-4-5A & 10.3 & 1.1 & 34.0 & 9,660 & $<6.04$ & $<0.46$ & 7.82 & 591 & $<116$ & 961 & 46.0 \\
\hline Z9-4-5A dup & 14.5 & 2.21 & 40.2 & 10,600 & 6.32 & $<0.86$ & 8.56 & $<883$ & $<214$ & 1,170 & 53.2 \\
\hline Z9-4-11A & 6.69 & $<0.66$ & 14.3 & 6,650 & 3.09 & $<0.71$ & 2.27 & $<734$ & $<178$ & 603 & 48.5 \\
\hline Z9-4-11A dup & NA & NA & NA & NA & NA & NA & NA & NA & NA & NA & NA \\
\hline B1HK-27 & 15.6 & $<0.51$ & 15.1 & 13,700 & 2.21 & 7.10 & $<1.4$ & $<561$ & $<136$ & 4,960 & 350 \\
\hline B1HK-27 dup & 9.91 & 0.599 & 9.96 & 11,800 & 2.70 & 1.92 & $<0.80$ & 698 & $<62$ & 3,050 & 159 \\
\hline B1HK-42 & 17.6 & 1.49 & 15.4 & 15,500 & 5.23 & 2.70 & $<1.9$ & 1,480 & $<187$ & 5,130 & 329 \\
\hline B1HK-42 dup & 18.7 & 2.83 & 12.8 & 14,300 & 4.97 & 2.45 & $<1.8$ & 1,380 & $<136$ & 5,150 & 305 \\
\hline Z12-1D & 200 & $<4.2$ & 54.3 & 52,700 & 11.5 & 6.08 & $<12$ & $<4,690$ & $<1,140$ & 7,570 & 610 \\
\hline Z12-1D dup & 193 & $<2.6$ & 29.6 & 53,500 & 10.7 & 5.25 & $<17$ & $<5,330$ & $<1,290$ & 7,230 & 613 \\
\hline
\end{tabular}

(continued). Z-9 and Z-12 sediment sample acid extraction results.

\begin{tabular}{|c|c|c|c|c|c|c|c|c|c|c|}
\hline Sample ID & ${ }^{97} \mathrm{Mo}(\mu \mathrm{g} / \mathrm{g})$ & $\mathrm{Na}^{\mathrm{ab}}(\mu \mathrm{g} / \mathrm{g})$ & ${ }^{60} \mathrm{Ni}(\mu \mathrm{g} / \mathrm{g})$ & $P(\mu \mathrm{g} / \mathrm{g})$ & ${ }^{208} \mathrm{~Pb}(\mu \mathrm{g} / \mathrm{g})$ & ${ }^{185} \operatorname{Re}(\mu \mathrm{g} / \mathrm{g})$ & ${ }^{101} \mathrm{Ru}(\mu \mathrm{g} / \mathrm{g})$ & $S(\mu \mathrm{g} / \mathrm{g})$ & ${ }^{121} \mathrm{Sb}(\mu \mathrm{g} / \mathrm{g})$ & ${ }^{82} \mathrm{Se}(\mu \mathrm{g} / \mathrm{g})$ \\
\hline Z9-4-5A & 43.1 & 451 & 4.02 & 5200 & 18.5 & $<0.63$ & $<0.61$ & 622 & 0.584 & $<4.9$ \\
\hline Z9-4-5A dup & 50.4 & $<650$ & 5.25 & 5590 & 20.0 & $<0.51$ & $<0.80$ & $<1,100$ & $<0.92$ & $<23$ \\
\hline Z9-4-11A & 14.9 & $<540$ & 2.19 & 931 & 41.7 & $<0.97$ & $<0.94$ & $<914$ & $<0.68$ & $<7.5$ \\
\hline Z9-4-11A dup & NA & NA & NA & NA & NA & NA & NA & NA & NA & NA \\
\hline B1HK-27 & $<1.5$ & 488 & 49.7 & 1,400 & 4.23 & $<0.74$ & $<0.71$ & 1,290 & $<0.52$ & $<5.7$ \\
\hline B1HK-27 dup & $<0.38$ & 257 & 10.3 & 716 & 5.34 & $<0.15$ & $<0.23$ & $<317$ & $<0.26$ & $<6.7$ \\
\hline B1HK-42 & $<2.1$ & $<567$ & 20.7 & 671 & 5.93 & $<1.0$ & $<0.98$ & $<959$ & $<0.72$ & $<7.9$ \\
\hline B1HK-42 dup & $<0.83$ & $<413$ & 21.0 & 706 & 5.53 & $<0.32$ & $<0.51$ & $<699$ & $<0.58$ & $<15$ \\
\hline Z12-1D & $<13$ & 7,750 & 146 & 2,010 & 48.1 & $<6.2$ & $<6.0$ & $<5,840$ & $<4.4$ & $<48$ \\
\hline Z12-1D dup & $<7.9$ & 7,940 & 136 & $<1,840$ & 43.1 & $<3.1$ & $<4.8$ & $<6,630$ & $<5.5$ & $<140$ \\
\hline
\end{tabular}


(continued). Z-9 and Z-12 sediment sample acid extraction results.

\begin{tabular}{ccccccccc}
\hline Sample ID & $\mathbf{S i}^{\mathbf{b}}(\boldsymbol{\mu g} \mathbf{g} \mathbf{g})$ & $\mathbf{S r}(\boldsymbol{\mu g} / \mathbf{g})$ & $\mathbf{2 3 2}^{\mathbf{T h}}(\boldsymbol{\mu \mathbf { g }} / \mathbf{g})$ & $\mathbf{T i}(\boldsymbol{\mu g} / \mathbf{g})$ & ${ }^{\mathbf{2 3 8}} \mathbf{U}(\boldsymbol{\mu g} / \mathbf{g})$ & $\mathbf{V}(\boldsymbol{\mu g} / \mathbf{g})$ & $\mathbf{Z n}(\boldsymbol{\mu g} / \mathbf{g})$ & $\mathbf{Z r}(\boldsymbol{\mu g} / \mathbf{g})$ \\
\hline Z9-4-5A & $<906$ & $<8.2$ & 0.831 & 513 & 20.3 & $<22$ & 80.7 \\
Z9-4-5A dup & $<1,670$ & $<15$ & 0.908 & 584 & 21.5 & $<41$ & 88.9 \\
Z9-4-11A & $<1,390$ & $<13$ & 0.577 & 632 & 5.75 & $<34$ & $<31$ \\
Z9-4-11A dup & NA & NA & NA & NA & NA & NA & NA & $<28$ \\
B1HK-27 & $<1,060$ & 13.2 & 2.43 & 457 & 50.2 & $<26$ & 45.5 & $<18$ \\
B1HK-27 dup & $<481$ & 13.4 & 1.97 & 448 & 1.56 & 15.7 & 29.7 & $<8.0$ \\
B1HK-42 & $<1,460$ & 26.0 & 4.11 & 650 & 0.982 & $<36$ & 43.2 \\
B1HK-42 dup & $<1,060$ & 26.3 & 3.33 & 558 & 0.893 & $<26$ & 37.6 & $<18$ \\
Z12-1D & $<8,860$ & 91.3 & 2.94 & 5,290 & 10.3 & $<219$ & $<200$ & $<147$ \\
Z12-1D dup & $<10,100$ & $<91$ & 3.02 & 5,000 & 10.0 & $<249$ & $<227$ & $<167$ \\
\hline * not analyzed & & & & & &
\end{tabular}

* not analyzed

a The concentrations measured in the preparatory blank were not $<$ EQL and were $>10 \%$ of the measured.

concentrations in the samples. This data does not meet Quality Control requirements and is For Information Only (FIO).

$\mathrm{b}$ The Blank Spike was not within $80-120 \%$ of the expected recovery. This data does not meet Quality Control requirements and is FIO. 
Table S4. Z-9 and Z-12 sediment groundwater extraction results for major and trace elements.

\begin{tabular}{|c|c|c|c|c|c|c|c|c|c|c|}
\hline Sample ID & ${ }^{107} \mathbf{A g}^{\mathrm{b}}(\mu \mathrm{g} / \mathrm{g})$ & $\mathrm{Al}(\mu \mathrm{g} / \mathrm{g})$ & ${ }^{75} \mathrm{As}(\mu \mathrm{g} / \mathrm{g})$ & B $(\mu \mathrm{g} / \mathrm{g})$ & ${ }^{138} \mathbf{B a}^{a}(\mu \mathrm{g} / g)$ & $\mathrm{Be}(\mu \mathrm{g} / \mathrm{g})$ & $\mathrm{Bi}(\mu \mathrm{g} / \mathrm{g})$ & $\mathrm{Ca}^{\mathrm{ab}}(\mu \mathrm{g} / \mathrm{g})$ & ${ }^{111} \mathrm{Cd}(\mu \mathrm{g} / \mathrm{g})$ & Co $(\mu \mathrm{g} / \mathrm{g})$ \\
\hline Z9-4-5A & 0.0577 & $<24$ & 0.125 & 435 & 0.110 & $<3.3$ & $<78$ & 1,090 & $<0.055$ & $<33$ \\
\hline Z9-4-5A dup & $<0.037$ & $<31$ & $<0.42$ & $<449$ & 0.130 & $<4.3$ & $<100$ & 650 & $<0.069$ & $<43$ \\
\hline Z9-4-11A & 0.145 & $<43$ & $<0.22$ & 660 & 0.558 & $<6.0$ & $<140$ & 975 & $<0.100$ & $<60$ \\
\hline Z9-4-11A dup & 0.226 & $<43$ & $<0.58$ & 732 & 0.426 & $<6.0$ & $<140$ & 917 & $<0.097$ & $<60$ \\
\hline B1HK-27 & $<0.022$ & $<10$ & $<0.053$ & 154 & 0.184 & $<1.5$ & $<34$ & 284 & 0.0813 & $<14$ \\
\hline B1HK-27 dup & $<0.007$ & $<5.8$ & $<0.078$ & 90.6 & 0.0923 & $<0.81$ & $<19$ & 154 & 0.047 & $<8.1$ \\
\hline B1HK-42 & $<0.028$ & $<13$ & $<0.066$ & 217 & 0.212 & $<1.8$ & $<42$ & 468 & 0.122 & $<18$ \\
\hline B1HK-42 dup & $<0.059$ & $<49$ & $<0.67$ & $<717$ & 0.623 & $<6.9$ & $<159$ & 1,060 & 0.159 & $<69$ \\
\hline Z12-1D & $<0.082$ & $<38$ & $<0.19$ & 697 & 0.133 & $<5.3$ & $<123$ & 375 & $<0.088$ & $<53$ \\
\hline Z12-1D dup & $<0.139$ & $<115$ & $<1.6$ & 1,700 & 0.633 & $<16$ & $<374$ & 2,070 & $<0.26$ & $<161$ \\
\hline
\end{tabular}

(continued). Z-9 and Z-12 sediment sample groundwater extraction results.

\begin{tabular}{|c|c|c|c|c|c|c|c|c|c|c|c|}
\hline Sample ID & $\begin{array}{c}{ }^{53} \mathrm{Cr} \\
(\mu \mathrm{g} / \mathrm{g})\end{array}$ & $\begin{array}{l}{ }^{133} \mathrm{Cs} \\
(\mu \mathrm{g} / \mathrm{g})\end{array}$ & $\begin{array}{c}{ }^{65} \mathrm{Cu} \\
(\mu \mathrm{g} / \mathrm{g})\end{array}$ & $\begin{array}{c}\mathbf{F e} \\
(\mu \mathrm{g} / \mathrm{g})\end{array}$ & $\begin{array}{c}{ }^{71} \mathbf{G a} \\
(\mu \mathrm{g} / \mathrm{g})\end{array}$ & $\begin{array}{l}{ }^{157} \mathbf{G d} \\
(\mu \mathrm{g} / \mathrm{g})\end{array}$ & $\begin{array}{l}{ }^{200} \mathrm{Hg} \\
(\mu \mathrm{g} / \mathrm{g})\end{array}$ & $\begin{array}{c}\mathbf{K}^{\mathbf{b}} \\
(\mu \mathrm{g} / \mathrm{g})\end{array}$ & $\begin{array}{c}\mathbf{L i} \\
(\mu \mathrm{g} / \mathrm{g})\end{array}$ & $\begin{array}{c}M^{\text {ab }} \\
(\mu \mathrm{g} / \mathrm{g})\end{array}$ & $\begin{array}{c}\text { Mn } \\
(\mu \mathrm{g} / \mathrm{g})\end{array}$ \\
\hline Z9-4-5A & 0.166 & $<0.036$ & 5.78 & $<27$ & $<0.038$ & $<0.038$ & 0.328 & $<329$ & $<190$ & 327 & $<16$ \\
\hline Z9-4-5A dup & 0.184 & 0.0308 & 2.88 & $<34$ & $<0.049$ & $<0.049$ & 0.266 & $<423$ & $<245$ & 333 & $<21$ \\
\hline Z9-4-11A & 0.243 & $<0.065$ & 0.415 & $<48$ & $<0.069$ & $<0.069$ & $<0.175$ & $<595$ & $<344$ & 345 & $<29$ \\
\hline Z9-4-11A dup & 0.259 & $<0.035$ & 0.486 & $<48$ & $<0.069$ & $<0.069$ & $<0.224$ & $<593$ & $<343$ & 331 & $<29$ \\
\hline B1HK-27 & 0.0617 & $<0.015$ & $<0.044$ & $<12$ & $<0.017$ & $<0.017$ & $<0.042$ & $<142$ & $<82$ & 85.9 & $<6.9$ \\
\hline B1HK-27 dup & $<0.027$ & $<0.0047$ & 0.0196 & $<6.5$ & $<0.0093$ & $<0.0093$ & $<0.030$ & $<80$ & $<46$ & 47.2 & $<3.9$ \\
\hline B1HK-42 & 0.0691 & $<0.019$ & $<0.055$ & $<14$ & $<0.021$ & $<0.021$ & $<0.052$ & $<176$ & $<102$ & 90.1 & $<8.5$ \\
\hline B1HK-42 dup & $<0.229$ & $<0.040$ & 0.163 & $<55$ & $<0.079$ & $<0.079$ & $<0.255$ & $<676$ & $<391$ & 285 & $<33$ \\
\hline Z12-1D & 0.213 & $<0.057$ & $<0.16$ & $<42$ & $<0.061$ & $<0.061$ & $<0.154$ & $<521$ & $<302$ & 171 & $<25$ \\
\hline Z12-1D dup & $<0.54$ & $<0.094$ & 0.330 & $<129$ & $<0.184$ & $<0.19$ & $<0.598$ & $<1,590$ & $<918$ & 763 & $<77$ \\
\hline
\end{tabular}

(continued). Z-9 and Z-12 sediment sample groundwater extraction results.

\begin{tabular}{|c|c|c|c|c|c|c|c|c|c|c|}
\hline Sample ID & $\begin{array}{c}{ }^{97} \mathrm{Mo} \\
(\mu \mathrm{g} / \mathrm{g})\end{array}$ & $\begin{array}{l}N^{\mathbf{a b}} \\
(\mu \mathrm{g} / \mathrm{g})\end{array}$ & $\begin{array}{c}{ }^{60} \mathrm{Ni} \\
(\mu \mathrm{g} / \mathrm{g})\end{array}$ & $\begin{array}{c}\mathbf{P} \\
(\mu \mathrm{g} / \mathrm{g})\end{array}$ & $\begin{array}{l}{ }^{208} \mathrm{~Pb} \\
(\mu \mathrm{g} / \mathrm{g})\end{array}$ & $\begin{array}{c}{ }^{185} \mathrm{Re} \\
(\mu \mathrm{g} / \mathrm{g})\end{array}$ & $\begin{array}{l}{ }^{101} \mathrm{Ru} \\
(\mu \mathrm{g} / \mathrm{g})\end{array}$ & $\begin{array}{c}\mathbf{S} \\
(\mu \mathrm{g} / \mathrm{g})\end{array}$ & $\begin{array}{c}{ }^{121} \mathrm{Sb} \\
(\mu \mathrm{g} / \mathrm{g})\end{array}$ & $\begin{array}{c}{ }^{82} \mathrm{Se}^{\mathrm{b}} \\
(\mu \mathrm{g} / \mathrm{g})\end{array}$ \\
\hline Z9-4-5A & 2.06 & 1,150 & 0.562 & 372 & $<0.039$ & $<0.052$ & $<0.050$ & $<540$ & $<0.037$ & $<0.40$ \\
\hline Z9-4-5A dup & 2.02 & 900 & 0.664 & 318 & $<0.023$ & $<0.029$ & $<0.046$ & $<695$ & $<0.053$ & $<1.33$ \\
\hline Z9-4-11A & 0.221 & 1,380 & 0.123 & $<278$ & $<0.070$ & $<0.094$ & $<0.091$ & $<976$ & $<0.067$ & $<0.73$ \\
\hline Z9-4-11A dup & 0.244 & 1,400 & 0.119 & $<277$ & $<0.032$ & $<0.041$ & $<0.065$ & $<974$ & $<0.074$ & $<1.87$ \\
\hline B1HK-27 & $<0.046$ & 354 & $<0.025$ & $<67$ & $<0.017$ & $<0.023$ & $<0.022$ & $<234$ & $<0.016$ & $<0.175$ \\
\hline B1HK-27 dup & $<0.014$ & 205 & $<0.014$ & $<37$ & $<0.0044$ & $<0.0055$ & $<0.0087$ & $<131$ & $<0.010$ & $<0.251$ \\
\hline B1HK-42 & $<0.057$ & 497 & $<0.031$ & $<82$ & $<0.021$ & $<0.028$ & $<0.027$ & $<290$ & $<0.020$ & $<0.217$ \\
\hline B1HK-42 dup & $<0.121$ & 1,460 & $<0.117$ & $<316$ & $<0.037$ & $<0.047$ & $<0.074$ & $<1,110$ & $<0.084$ & $<2.13$ \\
\hline Z12-1D & $<0.168$ & 2,490 & 0.173 & $<244$ & $<0.061$ & $<0.083$ & $<0.080$ & $<856$ & $<0.058$ & $<0.641$ \\
\hline Z12-1D dup & $<0.283$ & 4,990 & 0.331 & $<741$ & $<0.087$ & $<0.110$ & $<0.173$ & $<2,600$ & $<0.200$ & $<4.99$ \\
\hline
\end{tabular}


(continued). Z-9 and Z-12 sediment sample groundwater extraction results.

\begin{tabular}{|c|c|c|c|c|c|c|c|c|}
\hline Sample ID & $\begin{array}{c}\mathrm{Si}^{\mathbf{a b}} \\
(\mu \mathrm{g} / \mathrm{g})\end{array}$ & $\begin{array}{c}\mathrm{Sr} \\
(\mu \mathrm{g} / \mathrm{g})\end{array}$ & $\begin{array}{l}{ }^{232} \mathrm{Th} \\
(\mu \mathrm{g} / \mathrm{g})\end{array}$ & $\begin{array}{c}\mathbf{T i} \\
(\mu \mathrm{g} / \mathrm{g})\end{array}$ & $\begin{array}{c}\mathrm{U}^{\mathrm{a}} \\
(\mu \mathrm{g} / \mathrm{g})\end{array}$ & $\begin{array}{c}V \\
(\mu \mathrm{g} / \mathrm{g})\end{array}$ & $\begin{array}{c}\mathrm{Zn} \\
(\mu \mathrm{g} / \mathrm{g})\end{array}$ & $\begin{array}{c}\mathbf{Z r} \\
(\mu \mathrm{g} / \mathrm{g})\end{array}$ \\
\hline Z9-4-5A & 1,330 & $<20$ & $<0.0056$ & $<25$ & 0.105 & $<34$ & $<25$ & $<33$ \\
\hline Z9-4-5A dup & 1,440 & $<26$ & $<0.0072$ & $<33$ & 0.103 & $<44$ & $<32$ & $<42$ \\
\hline Z9-4-11A & 2,180 & $<37$ & $<0.0101$ & $<46$ & 0.0494 & $<61$ & $<45$ & $<59$ \\
\hline Z9-4-11A dup & 2,270 & $<37$ & $<0.0101$ & $<46$ & 0.0466 & $<61$ & $<45$ & $<59$ \\
\hline B1HK-27 & 508 & $<8.8$ & $<0.0024$ & $<11$ & 0.00372 & $<15$ & $<11$ & $<14$ \\
\hline B1HK-27 dup & 294 & $<5.0$ & $<0.0014$ & $<6.1$ & 0.00126 & $<8.2$ & $<6.0$ & $<7.9$ \\
\hline B1HK-42 & 671 & $<11$ & $<0.0040$ & $<14$ & 0.00305 & $<18$ & $<13$ & $<18$ \\
\hline B1HK-42 dup & 2,150 & $<42$ & $<0.0115$ & $<52$ & $<0.011$ & $<70$ & $<51$ & $<67$ \\
\hline Z12-1D & 2,010 & $<32$ & $<0.0088$ & $<40$ & 0.0702 & $<54$ & $<40$ & $<52$ \\
\hline Z12-1D dup & 5,440 & $<99$ & $<0.0269$ & $<122$ & 0.0749 & $<164$ & $<120$ & $<158$ \\
\hline
\end{tabular}

a The concentrations measured in the preparatory blank were not $<\mathrm{EQL}$ and were $>10 \%$ of the measured concentrations in the samples. This is likely due to the natural groundwater that was used for the extractions. This data does not meet Quality Control requirements and is For Information Only (FIO).

b The Blank Spike was not within $80-120 \%$ of the expected recovery. This is likely due to the natural groundwater that was used for the extractions (except for Ag). This data does not meet Quality Control requirements and is FIO. 


\subsection{Summary of extraction results SX-115 sediments}

Additional baseline sediments were collected from borehole 299-W23-19 (B8809) which was installed at the southwestern edge of tank SX-115 in 1999 using a reverse air-rotary method to a total depth of 75 meters (264 feet) [14]. The borehole intersected four principal stratigraphic layers: (1) backfill around high level waste tanks (up to $18.7 \mathrm{~m}$ or 61.5 feet), (2) the Hanford formation (18.7 - $37.2 \mathrm{~m}, 61.5$ - 122 feet), (3) the PiloPleistocene unit (37.2 - 48.8 m, 122 - 160 feet), and (4) terminating in the Ringold Unit E gravels 10.4 meters (34 feet) below the water table ( $67 \mathrm{~m}$ or $220 \mathrm{ft}$ ). Core samples from $>61.5$ feet $(18.7 \mathrm{~m})$ are included in these experiments in order to focus on Hanford sediments and exclude the backfill materials.

During drilling no radioactivity above background was detected. In addition, geophysical logs indicated that no man-made, radioactive contaminants were present with the exception of Cs-137 in the upper 1.5 meters. However, additional laboratory results showed that tank fluids have interacted with sediments between 65 and 156 feet bgs (19.8- $47.5 \mathrm{~m}$ ) based on $\mathrm{pH}$, conductivity, and major anion analyses. Tc-99 migrated deeper as concentrations above background are measured throughout the borehole suggesting some waste impacts throughout the borehole. Further, their comparison of contaminant ratios indicated that $\mathrm{NO}_{3}{ }^{-}, \mathrm{Tc}-99$, and Mo migrate without measurable retardation in the vadose zone while $\mathrm{Na}, \mathrm{Cr}$, and Se migrated with a small amount of retardation. Specifics for the depth for each sample are included in Table S5.

Both acid and groundwater extractions were conducted on these sediments as described in the Materials and Methods. Results are summarized in Tables S6-7 with all results included in Tables S8-9. Although there were no elements that were consistently elevated or depleted across all crib samples, the following elements were below detection limits for crib samples but measurable in the SX-115 sediments: $\mathrm{Al}, \mathrm{Be}, \mathrm{Fe}, \mathrm{Pb}, \mathrm{Th}, \mathrm{Zr}$.

Some significant differences were observed for both acid and groundwater extracts for the Z9 near-surface samples include (1) elevated $\mathrm{Cu}$ and Mo, (2) depleted $\mathrm{Al}$, and (3) Ag above detection limits (11.5 and $11.1 \mu \mathrm{g} / \mathrm{g}$ in Z9-5A and Z9-11A, respectively; not observed above detection limits in any other samples). However, it should also be noted 
that the only sample with measurable Bi was the acid extract for Z9-5A (332 $\pm 13 \mu \mathrm{g} / \mathrm{g}$ ). Further, for the Z9-11A and Z12-1D, samples were elevated in $\mathrm{Na}$ although this was not observed in Z9-5A due to significant error on duplicates (1025 \pm 178 versus $705 \pm 262$ $\mu \mathrm{g} / \mathrm{g}$ for Z9-5A and SX-115, respectively). Therefore, $\mathrm{Na}$ and trace elements including $\mathrm{Cu}, \mathrm{Mo}, \mathrm{Ag}$, and Bi may be used as indicators for the Pu-bearing waste based on these comparisons with near-surface crib samples.

The Z12-1D sediment was elevated in more heavy metals and exchangeable cations than the Z9 near-surface trench samples. For example, in the acid extractions, Al, exchangeable cations ( $\mathrm{Ba}, \mathrm{Cs}, \mathrm{Mg}, \mathrm{Mn}$, and $\mathrm{Na}$ ), and trace metals $(\mathrm{Cr}, \mathrm{Cu}, \mathrm{Ga}, \mathrm{Ni}, \mathrm{Pb}$, $\mathrm{Sr}$, $\mathrm{Ti}$, and $\mathrm{U}$ ) were elevated. Further, $\mathrm{Cd}$ and $\mathrm{Hg}$ were above detection limits in acid extractions for Z12 but not the SX-115 sediments. Although the Z12 crib received much lower ionic strength, neutral $\mathrm{pH}$ waste as compared to the $\mathrm{Z9}$ trench, it received significantly more volume $[10,15]$. Additional comparison of the deeper Z9 sediments from the slant borehole suggests elevated $\mathrm{Al}$ and $\mathrm{Ni}$ with $\mathrm{Cd}$ and $\mathrm{Hg}$ also below detection limits, adding several more waste indicators to the list.

Table S5. Z-9 and Z-12 sediment samples used for groundwater and acid extraction analyses.

\begin{tabular}{ccc}
\hline Sample ID & $\begin{array}{c}\text { Depth Below Ground } \\
\text { Surface (feet) }\end{array}$ & Description of Sample \\
\hline B8809-22E & $86.6-87.6$ & $299-W 23-19$, SX-115 \\
B8809-22D & $87.6-88.6$ & $299-W 23-19$, SX-115 \\
B8809-22C & $88.6-89.6$ & $299-W 23-19$, SX-115 \\
B8809-29H & $114.1-114.6$ & $299-W 23-19$, SX-115 \\
B8809-29G & $114.6-115.1$ & $299-W 23-19$, SX-115 \\
B8809-35E & $143.6-144.1$ & $299-W 23-19$, SX-115 \\
B8809-35D & $144.1-144.6$ & $299-W 23-19$, SX-115 \\
B8809-35C & $144.6-145.1$ & $299-W 23-19$, SX-115 \\
$B 8809-39 B$ & $156.1-156.6$ & $299-W 23-19, S X-115$ \\
$B 8809-39 A$ & $156.6-157.1$ & $299-W 23-19, S X-115$ \\
$B 8809-40 B$ & $157.5-158.0$ & $299-W 23-19, S X-115$ \\
\hline
\end{tabular}




\begin{tabular}{ccc}
\hline$B 8809-40 A$ & $158-158.5$ & $299-W 23-19, S X-115$ \\
$B 8809-40$ & $158.5-158.8$ & $299-W 23-19, S X-115$ \\
$B 8809-41$ & $184.9-185.2$ & $299-W 23-19, S X-115$ \\
$B 8809-42$ & $194.35-194.75$ & $299-W 23-19, S X-115$ \\
\hline
\end{tabular}

Table S6. Summary comparison of elements extracted in groundwater reacted with crib sediments with average of 16 sediments from the SX-115 borehole, Above DL - elements in cribs were above detection limits while elements were below detection limits in SX-115, Elevated - above SX-115 as compared for one standard deviation, Depleted - below SX115 as compared for one standard deviation, Below $\mathrm{DL}$ - below detection limits in crib sediments while measurable in SX-115 sediments.

\begin{tabular}{lllll}
\hline Sample ID & Above DL & Elevated & Depleted & Below DL \\
\hline Z9-5A & $\mathrm{Ag}, \mathrm{As}, \mathrm{Ni}, \mathrm{P}$ & $\mathrm{Cu}, \mathrm{Mg}, \mathrm{Mo}$ & $\mathrm{Ba}$ & $\mathrm{Al}, \mathrm{Be}, \mathrm{Fe}, \mathrm{Hg}, \mathrm{Pb}, \mathrm{Th}, \mathrm{Zr}$ \\
Z9-11A & $\mathrm{Ag}, \mathrm{Ni}$ & $\mathrm{Ca}, \mathrm{Cu}, \mathrm{Mg}, \mathrm{Mo}, \mathrm{Na}$ & & $\mathrm{Al}, \mathrm{Be}, \mathrm{Cs}, \mathrm{Fe}, \mathrm{Pb}, \mathrm{Th}, \mathrm{Zr}$ \\
$\mathrm{B} 1 \mathrm{HK}-27$ & $\mathrm{Cd}$ & $\mathrm{Ba}$ & $\mathrm{Al}, \mathrm{Be}, \mathrm{Cs}, \mathrm{Fe}, \mathrm{Mo}, \mathrm{Pb}, \mathrm{Th}, \mathrm{Zr}$ \\
$\mathrm{B} 1 \mathrm{HK}-42$ & $\mathrm{Cd}$ & & $\mathrm{Al}, \mathrm{Be}, \mathrm{Cs}, \mathrm{Fe}, \mathrm{Mo}, \mathrm{Pb}, \mathrm{Th}, \mathrm{Zr}$ \\
Z12-1D & $\mathrm{Ni}$ & $\mathrm{Na}$ & & $\mathrm{Al}, \mathrm{Be}, \mathrm{Cs}, \mathrm{Fe}, \mathrm{Mo}, \mathrm{Pb}, \mathrm{Th}, \mathrm{Zr}$ \\
\hline
\end{tabular}

Table S7. Summary comparison of elements extracted in acid with crib sediments with average of 16 sediments from the SX-115 borehole, Above DL - elements in cribs were above detection limits while elements were below detection limits in SX-115, Elevated above SX-115 as compared for one standard deviation, Depleted - below SX-115 as compared for one standard deviation, Below $\mathrm{DL}$ - below detection limits in crib sediments while measurable in SX-115 sediments.

\begin{tabular}{lllll}
\hline Sample ID & Above DL & Elevated & Depleted & Below DL \\
\hline Z9-5A & $\mathrm{Ag}, \mathrm{Bi}, \mathrm{Hg}, \mathrm{Sb}$ & $\mathrm{Cu}, \mathrm{Mo}, \mathrm{P}, \mathrm{Pb}, \mathrm{U}, \mathrm{Zn}$ & $\mathrm{Al}, \mathrm{Ba}, \mathrm{K}, \mathrm{Mg}, \mathrm{Mn}, \mathrm{Ni}$ & $\mathrm{Sr}, \mathrm{V}, \mathrm{Zr}$ \\
$\mathrm{Z9-11 \textrm {A }}$ & $\mathrm{Ag}, \mathrm{Hg}$ & $\mathrm{Mo}, \mathrm{Pb}, \mathrm{U}$ & $\mathrm{Al}, \mathrm{Ba}, \mathrm{K}, \mathrm{Mg}, \mathrm{Mn}, \mathrm{Ni}$ & $\mathrm{As}, \mathrm{K}, \mathrm{Na}, \mathrm{Sr}, \mathrm{V}, \mathrm{Zn}, \mathrm{Zr}$ \\
$\mathrm{B} 1 \mathrm{HK}-27$ & $\mathrm{Cd}, \mathrm{Hg}$ & $\mathrm{Al}, \mathrm{Ni}, \mathrm{U}$ & $\mathrm{Ba}, \mathrm{K}$ & $\mathrm{As}, \mathrm{Mo}, \mathrm{Na}, \mathrm{Sr}, \mathrm{Zr}$ \\
$\mathrm{B} 1 \mathrm{HK}-42$ & $\mathrm{Cd}, \mathrm{Hg}$ & $\mathrm{Al}, \mathrm{Cs}, \mathrm{Ni}$ & $\mathrm{Mo}, \mathrm{Na}, \mathrm{V}, \mathrm{Zr}$ \\
& & $\mathrm{Al}, \mathrm{Ba}, \mathrm{Cr}, \mathrm{Cs}, \mathrm{Cu}, \mathrm{Fe}, \mathrm{Ga}, \mathrm{Mg}$, & & $\mathrm{As}, \mathrm{K}, \mathrm{Mo}, \mathrm{V}, \mathrm{Zn}, \mathrm{Zr}$ \\
\hline
\end{tabular}

Table S8. SX-115 sediment sample acid extraction results.

\begin{tabular}{lccccccccc}
\hline Sample ID & ${ }^{107} \mathrm{Ag}$ & $\mathrm{Al}$ & ${ }^{75} \mathrm{As}$ & ${ }^{138} \mathrm{Ba}$ & $\mathrm{Be}$ & $\mathrm{Bi}$ & $\mathrm{Ca}$ & ${ }^{111} \mathrm{Cd}$ & $\mathrm{Co}$ \\
$(\mu \mathrm{g} / \mathrm{g})$ & $(\mu \mathrm{g} / \mathrm{g})$ & $(\mu \mathrm{g} / \mathrm{g})$ & $(\mu \mathrm{g} / \mathrm{g})$ & $(\mu \mathrm{g} / \mathrm{g})$ & $\begin{array}{c}\mathrm{Ca} / \mathrm{g}) \\
(\mu \mathrm{g} / \mathrm{g})\end{array}$ & $\begin{array}{c}(\mu \mathrm{g} / \mathrm{g}) \\
(\mu \mathrm{g} / \mathrm{g})\end{array}$ \\
\hline
\end{tabular}




\begin{tabular}{|c|c|c|c|c|c|c|c|c|c|}
\hline B8809-17H & $<0.40$ & 7,160 & $<4.5$ & 65.8 & $<4.9$ & $<61$ & 8,160 & $<0.75$ & $<31$ \\
\hline B8809-17H dup & $<0.44$ & 6,920 & $<5.0$ & 65.3 & $<5.4$ & $<67$ & 7,820 & $<0.83$ & $<34$ \\
\hline B8809-22C & $<0.49$ & 5,550 & $<5.5$ & 63.4 & $<6.0$ & $<74$ & 8,570 & $<0.91$ & $<38$ \\
\hline B8809-22C dup & $<0.47$ & 5,520 & $<5.2$ & 62.6 & $<5.7$ & $<70$ & 7,770 & $<0.86$ & $<36$ \\
\hline B8809-22D & $<0.56$ & 5,890 & $<6.3$ & 62.1 & $<6.9$ & $<85$ & 8,570 & $<1.04$ & $<43$ \\
\hline B8809-22D dup & $<0.38$ & 5,730 & $<4.3$ & 61.8 & $<4.7$ & $<57$ & 8,740 & $<0.71$ & $<29$ \\
\hline B8809-22E & $<0.42$ & 4,530 & $<4.7$ & 52.1 & $<5.1$ & $<63$ & 11,200 & $<0.78$ & $<32$ \\
\hline B8809-22E dup & $<0.45$ & 4,700 & $<5.0$ & 59.3 & $<5.5$ & $<67$ & 8,100 & $<0.83$ & $<34$ \\
\hline B8809-29G & $<0.38$ & 5,320 & $<4.3$ & 64.1 & $<4.7$ & $<58$ & 9,570 & $<0.71$ & $<29$ \\
\hline B8809-29G dup & $<0.58$ & 5,320 & $<6.5$ & 65.2 & $<7.0$ & $<87$ & 10,400 & $<1.07$ & $<44$ \\
\hline В8809-29H & $<0.39$ & 5,530 & $<4.4$ & 75.1 & $<4.8$ & $<59$ & 10,400 & $<0.73$ & $<30$ \\
\hline B8809-29H dup & $<0.41$ & 5,500 & $<4.6$ & 71.9 & $<5.1$ & $<62$ & 9,690 & $<0.77$ & $<32$ \\
\hline B8809-35C & $<0.47$ & 8,010 & $<5.3$ & 127 & $<5.7$ & $<71$ & 11,100 & $<0.87$ & $<36$ \\
\hline B8809-35C dup & $<0.37$ & 6,280 & $<4.1$ & 82.1 & $<4.5$ & $<55$ & 11,600 & $<0.68$ & $<28$ \\
\hline B8809-35D & $<0.24$ & 8,610 & 3.53 & 113 & $<2.9$ & $<36$ & 14,200 & $<0.44$ & $<18$ \\
\hline B8809-35D dup & $<0.23$ & 8,530 & 3.06 & 102 & $<2.8$ & $<34$ & 13,400 & $<0.42$ & $<17$ \\
\hline B8809-35E & $<0.31$ & 9,890 & 3.86 & 126 & $<3.8$ & $<46$ & 17,600 & $<0.57$ & $<24$ \\
\hline B8809-35E dup & $<0.37$ & 10,600 & 4.5 & 134 & $<4.5$ & $<55$ & 18,100 & $<0.68$ & $<28$ \\
\hline B8809-39A & $<0.26$ & 5,710 & 5.75 & 122 & $<3.2$ & $<39$ & 40,900 & $<0.49$ & $<20$ \\
\hline B8809-39A dup & $<0.35$ & 6,730 & 7.95 & 129 & $<4.2$ & $<52$ & 33,300 & $<0.64$ & $<26$ \\
\hline B8809-39B & $<0.27$ & 4,020 & 5.19 & 109 & $<3.2$ & $<40$ & 15,900 & $<0.49$ & $<20$ \\
\hline B8809-39B dup & $<0.30$ & 4,380 & 5.57 & 112 & $<3.6$ & $<45$ & 17,100 & $<0.55$ & $<23$ \\
\hline B8809-40 & $<0.45$ & 5,470 & $<5.0$ & 134 & $<5.5$ & $<68$ & 101,000 & $<0.84$ & $<34$ \\
\hline B8809-40 dup & $<0.40$ & 4,600 & 5.84 & 151 & $<4.9$ & $<60$ & 83,000 & $<0.75$ & $<31$ \\
\hline B8809-40A & $<0.43$ & 4,480 & $<4.9$ & 90.2 & $<5.3$ & $<65$ & 12,000 & $<0.81$ & $<33$ \\
\hline B8809-40A dup & $<0.37$ & 4,720 & 4.39 & 94.0 & $<4.6$ & $<56$ & 13,800 & $<0.69$ & $<29$ \\
\hline B8809-40B & $<0.29$ & 4,390 & 4.41 & 84.6 & $<3.6$ & $<44$ & 9,620 & $<0.55$ & $<23$ \\
\hline B8809-40B dup & $<0.25$ & 4,310 & 3.68 & 82.4 & $<3.0$ & $<37$ & 12,500 & $<0.46$ & $<19$ \\
\hline B8809-41 & $<0.39$ & 3,790 & $<4.4$ & 36.6 & $<4.8$ & $<59$ & 1,780 & $<0.73$ & $<30$ \\
\hline B8809-41 dup & $<0.53$ & 3,760 & $<5.9$ & 36.7 & $<6.4$ & $<79$ & 1,640 & $<0.98$ & $<40$ \\
\hline B8809-42 & $<0.42$ & 5,490 & $<4.7$ & 48.4 & $<5.1$ & $<63$ & 2,650 & $<0.78$ & $<32$ \\
\hline B8809-42 dup & $<0.46$ & 6,030 & $<5.1$ & 69.3 & $<5.6$ & $<69$ & 2,950 & $<0.85$ & $<35$ \\
\hline
\end{tabular}


Table S8 (continued). SX-115 sediment sample acid extraction results.

\begin{tabular}{|c|c|c|c|c|c|c|c|c|c|c|}
\hline Sample ID & $\begin{array}{c}{ }^{52} \mathrm{Cr} \\
(\mu \mathrm{g} / \mathrm{g})\end{array}$ & $\begin{array}{l}{ }^{133} \mathrm{Cs} \\
(\mu \mathrm{g} / \mathrm{g})\end{array}$ & $\begin{array}{l}{ }^{65} \mathrm{Cu} \\
(\mu \mathrm{g} / \mathrm{g})\end{array}$ & $\begin{array}{c}\mathrm{Fe} \\
(\mu \mathrm{g} / \mathrm{g})\end{array}$ & $\begin{array}{c}{ }^{69} \mathrm{Ga} \\
(\mu \mathrm{g} / \mathrm{g})\end{array}$ & $\begin{array}{r}{ }^{157} \mathrm{Gd} \\
(\mu \mathrm{g} / \mathrm{g})\end{array}$ & $\begin{array}{l}{ }^{200} \mathrm{Hg} \\
(\mu \mathrm{g} / \mathrm{g})\end{array}$ & $\begin{array}{c}\mathrm{K} \\
(\mu \mathrm{g} / \mathrm{g})\end{array}$ & $\begin{array}{c}\mathrm{Mg} \\
(\mu \mathrm{g} / \mathrm{g})\end{array}$ & $\begin{array}{c}\mathrm{Mn} \\
(\mu \mathrm{g} / \mathrm{g})\end{array}$ \\
\hline B8809-17H & 9.46 & 0.748 & 11.4 & 14,500 & 4.54 & 1.51 & $<1.7$ & 1,160 & 4,640 & 330 \\
\hline B8809-17H dup & 9.42 & 0.756 & 12.9 & 14,000 & 4.49 & 1.58 & $<1.9$ & 1,140 & 4,570 & 336 \\
\hline B8809-22C & 16.7 & 1.24 & 10.5 & 12,500 & 4.06 & 1.77 & $<2.1$ & 1,160 & 4,590 & 314 \\
\hline B8809-22C dup & 17.3 & 1.27 & 10.8 & 12,500 & 3.89 & 1.76 & $<2.0$ & 1,170 & 4,700 & 287 \\
\hline B8809-22D & 29.3 & 1.45 & 11.5 & 12,500 & 4.12 & 1.88 & $<2.4$ & 1,330 & 4,700 & 280 \\
\hline B8809-22D dup & 27.8 & 1.33 & 10.9 & 11,800 & 3.93 & 1.69 & $<1.6$ & 1,210 & 4,620 & 278 \\
\hline B8809-22E & 14.5 & 0.876 & 10.6 & 11,200 & 3.20 & 1.60 & $<1.8$ & 1,010 & 3,890 & 224 \\
\hline B8809-22E dup & 17.1 & 1.01 & 9.71 & 12,000 & 3.48 & 1.69 & $<1.9$ & 1,040 & 4,020 & 266 \\
\hline B8809-29G & 19.7 & 1.20 & 9.06 & 11,200 & 4.05 & 1.53 & $<1.7$ & 1,320 & 4,210 & 275 \\
\hline B8809-29G dup & 20.1 & 1.11 & 9.15 & 11,600 & 4.13 & 2.00 & $<2.5$ & 1,220 & 4,360 & 282 \\
\hline В8809-29H & 21.1 & 1.17 & 9.94 & 11,600 & 4.27 & 1.60 & $<1.7$ & 1,320 & 4,380 & 287 \\
\hline B8809-29H dup & 19.9 & 1.21 & 9.30 & 11,300 & 4.31 & 1.81 & $<1.8$ & 1,340 & 4,240 & 289 \\
\hline B8809-35C & 16.8 & 2.15 & 15.3 & 15,800 & 7.01 & 1.93 & $<2.0$ & 2,180 & 6,620 & 345 \\
\hline B8809-35C dup & 13.4 & 1.39 & 12.5 & 13,300 & 4.87 & 1.75 & $<1.6$ & 1,390 & 5,660 & 233 \\
\hline B8809-35D & 15.0 & 1.83 & 17.9 & 16,900 & 6.27 & 2.42 & $<1.0$ & 1,760 & 6,830 & 416 \\
\hline B8809-35D dup & 15.0 & 1.85 & 16.6 & 16,000 & 5.96 & 2.27 & $<0.98$ & 1,860 & 6,640 & 367 \\
\hline B8809-35E & 15.4 & 1.94 & 22.3 & 19,500 & 7.28 & 3.09 & $<1.3$ & 1,940 & 7,820 & 579 \\
\hline B8809-35E dup & 16.9 & 2.11 & 23.8 & 20,700 & 7.33 & 3.14 & $<1.6$ & 2,040 & 8,300 & 574 \\
\hline B8809-39A & 5.59 & 0.646 & 14.4 & 18,100 & 5.25 & 5.26 & $<1.1$ & 1,070 & 4,560 & 453 \\
\hline B8809-39A dup & 6.55 & 0.902 & 17.1 & 21,800 & 5.99 & 5.37 & $<1.5$ & 1,320 & 5,190 & 542 \\
\hline B8809-39B & 2.48 & 0.227 & 12.1 & 19,500 & 4.46 & 5.85 & $<1.1$ & 771 & 3,040 & 282 \\
\hline B8809-39B dup & 3.23 & 0.287 & 11.6 & 20,000 & 4.63 & 5.79 & $<1.3$ & 854 & 3,180 & 321 \\
\hline B8809-40 & 5.03 & 0.330 & 13.4 & 13,600 & 5.54 & 4.00 & $<1.9$ & 793 & 4,140 & 1,000 \\
\hline B8809-40 dup & 4.28 & 0.293 & 13.6 & 14,300 & 5.66 & 4.41 & $<1.7$ & 851 & 3,560 & 973 \\
\hline B8809-40A & 4.58 & 0.301 & 15.1 & 18,400 & 4.27 & 5.42 & $<1.9$ & 781 & 2,860 & 259 \\
\hline B8809-40A dup & 4.39 & 0.291 & 12.1 & 17,900 & 4.26 & 5.34 & $<1.6$ & 805 & 2,970 & 285 \\
\hline B8809-40B & 4.43 & 0.294 & 11.8 & 18,100 & 3.99 & 4.89 & $<1.3$ & 744 & 2,690 & 259 \\
\hline B8809-40B dup & 3.99 & 0.274 & 10.6 & 16,100 & 3.86 & 4.68 & $<1.1$ & 710 & 2,640 & 274 \\
\hline B8809-41 & 7.67 & 0.666 & 10.3 & 8,080 & 2.79 & 42.7 & $<1.7$ & 903 & 2,690 & 186 \\
\hline B8809-41 dup & 8.08 & 0.683 & 11.4 & 7,910 & 2.60 & 81.0 & $<2.3$ & 875 & 2,610 & 188 \\
\hline B8809-42 & 11.6 & 1.12 & 18.3 & 11,400 & 4.07 & 72.7 & $<1.8$ & 1,270 & 4,070 & 264 \\
\hline B8809-42 dup & 13.2 & 1.38 & 18.9 & 13,200 & 4.82 & 198 & $<2.0$ & 1,640 & 4,570 & 319 \\
\hline
\end{tabular}


Table S8 (continued). SX-115 sediment sample acid extraction results.

\begin{tabular}{|c|c|c|c|c|c|c|c|c|c|c|}
\hline Sample ID & $\begin{array}{l}{ }^{95} \mathrm{Mo} \\
(\mu \mathrm{g} / \mathrm{g})\end{array}$ & $\begin{array}{c}\mathrm{Na} \\
(\mu \mathrm{g} / \mathrm{g})\end{array}$ & $\begin{array}{c}{ }^{60} \mathrm{Ni} \\
(\mu \mathrm{g} / \mathrm{g})\end{array}$ & $\begin{array}{c}\mathrm{P} \\
(\mu \mathrm{g} / \mathrm{g})\end{array}$ & $\begin{array}{l}{ }^{206} \mathrm{~Pb} \\
(\mu \mathrm{g} / \mathrm{g})\end{array}$ & $\begin{array}{l}{ }^{185} \mathrm{Re} \\
(\mu \mathrm{g} / \mathrm{g})\end{array}$ & $\begin{array}{c}{ }^{101} \mathrm{Ru} \\
(\mu \mathrm{g} / \mathrm{g})\end{array}$ & $\begin{array}{c}\mathrm{S} \\
(\mu \mathrm{g} / \mathrm{g})\end{array}$ & $\begin{array}{c}{ }^{121} \mathrm{Sb} \\
(\mu \mathrm{g} / \mathrm{g})\end{array}$ & $\begin{array}{c}{ }^{82} \mathrm{Se} \\
(\mu \mathrm{g} / \mathrm{g}) \\
\end{array}$ \\
\hline B8809-17H & $<0.50$ & 536 & 10.3 & 560 & 2.94 & $<0.32$ & $<0.50$ & $<688$ & $<0.57$ & $<15$ \\
\hline B8809-17H dup & $<0.63$ & 593 & 11.0 & 591 & 3.70 & $<0.35$ & $<0.55$ & $<756$ & $<0.63$ & $<16$ \\
\hline B8809-22C & $<0.69$ & 953 & 11.9 & 669 & 5.40 & $<0.39$ & $<0.61$ & $<836$ & $<0.70$ & $<18$ \\
\hline B8809-22C dup & $<0.66$ & 1070 & 12.5 & 536 & 5.42 & $<0.37$ & $<0.58$ & $<792$ & $<0.66$ & $<17$ \\
\hline B8809-22D & $<0.79$ & 1470 & 13.3 & 700 & 5.46 & $<0.44$ & $<0.70$ & $<956$ & $<0.80$ & $<20$ \\
\hline B8809-22D dup & $<0.54$ & 1380 & 13.1 & 685 & 5.25 & $<0.30$ & $<0.47$ & $<649$ & $<0.54$ & $<14$ \\
\hline B8809-22E & $<0.59$ & 1040 & 10.2 & 597 & 3.41 & $<0.33$ & $<0.52$ & $<714$ & $<0.59$ & $<15$ \\
\hline B8809-22E dup & $<0.63$ & 1140 & 8.49 & 582 & 3.86 & $<0.35$ & $<0.55$ & $<760$ & $<0.63$ & $<16$ \\
\hline B8809-29G & $<0.54$ & 635 & 8.51 & 578 & 5.07 & $<0.30$ & $<0.48$ & $<654$ & $<0.54$ & $<14$ \\
\hline B8809-29G dup & $<0.81$ & 900 & 9.14 & 630 & 4.92 & $<0.46$ & $<0.72$ & $<982$ & $<0.82$ & $<21$ \\
\hline B8809-29H & $<0.56$ & 559 & 9.07 & 558 & 4.87 & $<0.31$ & $<0.49$ & $<671$ & $<0.56$ & $<14$ \\
\hline B8809-29H dup & $<0.58$ & 674 & 8.88 & 545 & 4.59 & $<0.33$ & $<0.51$ & $<704$ & $<0.59$ & $<15$ \\
\hline B8809-35C & $<0.66$ & 657 & 17.2 & 580 & 8.38 & $<0.37$ & $<0.58$ & $<798$ & $<0.66$ & $<17$ \\
\hline B8809-35C dup & $<0.52$ & 579 & 12.8 & 662 & 6.36 & $<0.29$ & $<0.46$ & $<624$ & $<0.52$ & $<13$ \\
\hline B8809-35D & $<0.34$ & 413 & 16.3 & 705 & 10.2 & $<0.19$ & $<0.30$ & $<406$ & $<0.34$ & $<8.5$ \\
\hline B8809-35D dup & $<0.32$ & 447 & 15.8 & 671 & 9.17 & $<0.18$ & $<0.28$ & $<388$ & $<0.32$ & $<8.2$ \\
\hline B8809-35E & $<0.43$ & 566 & 18.4 & 722 & 12.5 & $<0.24$ & $<0.38$ & $<524$ & $<0.44$ & $<11$ \\
\hline B8809-35E dup & $<0.52$ & 619 & 21.1 & 707 & 13.7 & $<0.29$ & $<0.46$ & $<625$ & $<0.52$ & $<13$ \\
\hline B8809-39A & $<0.37$ & 539 & 10.4 & 1,760 & 5.36 & $<0.21$ & $<0.33$ & $<445$ & $<0.37$ & $<9.4$ \\
\hline B8809-39A dup & $<0.49$ & 632 & 12.3 & 1,800 & 6.60 & $<0.27$ & $<0.43$ & $<589$ & $<0.49$ & $<12$ \\
\hline B8809-39B & $<0.37$ & 565 & 6.18 & 2,090 & 3.40 & $<0.21$ & $<0.33$ & $<451$ & $<0.38$ & $<9.5$ \\
\hline B8809-39B dup & $<0.42$ & 580 & 6.51 & 2,140 & 3.61 & $<0.24$ & $<0.37$ & $<507$ & $<0.42$ & $<11$ \\
\hline B8809-40 & $<0.63$ & 883 & 10.7 & 1,560 & 3.27 & $<0.36$ & $<0.56$ & $<767$ & $<0.64$ & $<16$ \\
\hline B8809-40 dup & $<0.57$ & 598 & 9.11 & 1,870 & 3.09 & $<0.32$ & $<0.50$ & $<683$ & $<0.57$ & $<14$ \\
\hline B8809-40A & $<0.61$ & 702 & 8.50 & 1,870 & 3.46 & $<0.34$ & $<0.54$ & $<740$ & $<0.62$ & $<16$ \\
\hline B8809-40A dup & $<0.53$ & 634 & 7.33 & 1,910 & 3.68 & $<0.30$ & $<0.46$ & $<635$ & $<0.53$ & $<13$ \\
\hline B8809-40B & 0.545 & 543 & 6.87 & 1,740 & 3.25 & $<0.23$ & $<0.37$ & $<501$ & $<0.42$ & $<11$ \\
\hline B8809-40B dup & $<0.35$ & 493 & 6.52 & 1,720 & 3.27 & $<0.20$ & $<0.31$ & $<423$ & $<0.35$ & $<8.9$ \\
\hline B8809-41 & $<0.56$ & 524 & 11.1 & 344 & 2.74 & $<0.31$ & $<0.49$ & $<672$ & $<0.56$ & $<14$ \\
\hline B8809-41 dup & $<0.74$ & 591 & 10.8 & 311 & 2.35 & $<0.42$ & $<0.66$ & $<898$ & $<0.75$ & $<19$ \\
\hline B8809-42 & 0.679 & 490 & 19.3 & 490 & 2.98 & $<0.33$ & $<0.52$ & $<715$ & $<0.60$ & $<15$ \\
\hline B8809-42 dup & 0.897 & 569 & 17.2 & 590 & 3.20 & $<0.36$ & $<0.57$ & $<778$ & $<0.65$ & $<16$ \\
\hline
\end{tabular}


Table S8 (continued). SX-115 sediment sample acid extraction results.

\begin{tabular}{|c|c|c|c|c|c|c|c|c|}
\hline Sample ID & $\begin{array}{c}\mathrm{Si} \\
(\mu \mathrm{g} / \mathrm{g}) \\
\end{array}$ & $\begin{array}{c}\mathrm{Sr} \\
(\mu \mathrm{g} / \mathrm{g})\end{array}$ & $\begin{array}{l}{ }^{232} \mathrm{Th} \\
(\mu \mathrm{g} / \mathrm{g})\end{array}$ & $\begin{array}{c}\mathrm{Ti} \\
(\mu \mathrm{g} / \mathrm{g}) \\
\end{array}$ & $\begin{array}{c}\mathrm{U} \\
(\mu \mathrm{g} / \mathrm{g}) \\
\end{array}$ & $\begin{array}{c}\mathrm{V} \\
(\mu \mathrm{g} / \mathrm{g}) \\
\end{array}$ & $\begin{array}{c}\mathrm{Zn} \\
(\mu \mathrm{g} / \mathrm{g}) \\
\end{array}$ & $\begin{array}{c}\mathrm{Zr} \\
(\mu \mathrm{g} / \mathrm{g}) \\
\end{array}$ \\
\hline B8809-17H & $<1040$ & 34.6 & 1.56 & 516 & 0.276 & 26.1 & 34.3 & $<17$ \\
\hline B8809-17H dup & $<1150$ & 38.4 & 1.91 & 464 & 0.282 & $<28$ & 33.8 & $<19$ \\
\hline B8809-22C & $<1270$ & 30.5 & 3.95 & 383 & 0.448 & $<31$ & 30.4 & $<21$ \\
\hline B8809-22C dup & $<1200$ & 27.8 & 27.8 & 369 & 1.40 & $<30$ & 33.3 & $<20$ \\
\hline B8809-22D & $<1450$ & 29.8 & 4.10 & 389 & 0.486 & $<36$ & $<33$ & $<24$ \\
\hline B8809-22D dup & $<985$ & 29.2 & 3.55 & 325 & 0.493 & $<24$ & 30.7 & $<16$ \\
\hline B8809-22E & $<1080$ & 35.5 & 2.42 & 370 & 0.392 & $<27$ & 26.1 & $<18$ \\
\hline B8809-22E dup & $<1150$ & 31.7 & 2.52 & 409 & 0.380 & $<29$ & 27.3 & $<19$ \\
\hline B8809-29G & $<992$ & 27.9 & 3.11 & 433 & 0.398 & $<25$ & 30.8 & $<16$ \\
\hline B8809-29G dup & $<1490$ & 29.6 & 3.37 & 433 & 0.612 & $<37$ & $<34$ & $<25$ \\
\hline В8809-29H & $<1020$ & 32.3 & 3.54 & 443 & 0.811 & $<25$ & 29.9 & $<17$ \\
\hline B8809-29H dup & $<1070$ & 34.7 & 3.13 & 442 & 0.408 & $<26$ & 27.5 & $<18$ \\
\hline B8809-35C & $<1210$ & 37.8 & 3.95 & 616 & 0.599 & $<30$ & 49.1 & $<20$ \\
\hline B8809-35C dup & $<948$ & 34.3 & 3.57 & 404 & 0.546 & $<23$ & 35.6 & $<16$ \\
\hline B8809-35D & $<616$ & 41.0 & 4.50 & 438 & 0.703 & 21.8 & 49.3 & $<10$ \\
\hline B8809-35D dup & $<589$ & 39.3 & 4.62 & 451 & 0.680 & 20.7 & 46 & $<9.8$ \\
\hline B8809-35E & $<795$ & 46.3 & 6.03 & 441 & 0.973 & 23.9 & 57 & $<13$ \\
\hline B8809-35E dup & $<949$ & 47.9 & 6.47 & 492 & 1.05 & 31.2 & 60 & $<16$ \\
\hline B8809-39A & $<676$ & 59.1 & 2.83 & 666 & 1.13 & 24.1 & 30.8 & 18.0 \\
\hline B8809-39A dup & $<894$ & 48.8 & 3.46 & 726 & 1.10 & 32.5 & 36.2 & 17.1 \\
\hline B8809-39B & $<685$ & 31.2 & 2.07 & 764 & 0.589 & 27.9 & 25.8 & 29.1 \\
\hline B8809-39B dup & $<769$ & 33.3 & 2.09 & 832 & 0.656 & 30.4 & 28 & 28.4 \\
\hline B8809-40 & $<1160$ & 100 & 1.84 & 653 & 5.10 & $<29$ & $<26$ & 20.8 \\
\hline B8809-40 dup & $<1040$ & 83.3 & 1.91 & 591 & 4.76 & $<26$ & $<23$ & 22.4 \\
\hline B8809-40A & $<1120$ & 28.5 & 2.19 & 846 & 0.728 & 33.3 & 26.2 & 26.9 \\
\hline B8809-40A dup & $<963$ & 32.2 & 2.22 & 810 & 0.811 & 33.7 & 25.5 & 25.3 \\
\hline B8809-40B & $<760$ & 24.2 & 2.10 & 754 & 0.631 & 31.9 & 28.5 & 23.7 \\
\hline B8809-40B dup & $<642$ & 27.8 & 1.90 & 608 & 0.700 & 28.6 & 25.9 & 24.2 \\
\hline B8809-41 & $<1020$ & 10.4 & 2.71 & 278 & 0.296 & $<25$ & $<23$ & $<17$ \\
\hline B8809-41 dup & $<1360$ & 13.4 & 2.00 & 263 & 0.287 & $<34$ & $<31$ & $<23$ \\
\hline B8809-42 & $<1080$ & 12.8 & 1.93 & 479 & 0.386 & $<27$ & 33.2 & $<18$ \\
\hline B8809-42 dup & $<1180$ & 14.7 & 2.10 & 702 & 0.417 & $<29$ & 39.1 & $<20$ \\
\hline
\end{tabular}


Table S9 SX-115 sediment sample groundwater extraction results.

\begin{tabular}{|c|c|c|c|c|c|c|c|c|c|}
\hline Sample ID & $\begin{array}{l}{ }^{107} \mathrm{Ag} \\
(\mu \mathrm{g} / \mathrm{g})\end{array}$ & $\begin{array}{c}\mathrm{Al} \\
(\mu \mathrm{g} / \mathrm{g})\end{array}$ & $\begin{array}{c}{ }^{75} \mathrm{As} \\
(\mu \mathrm{g} / \mathrm{g})\end{array}$ & $\begin{array}{l}{ }^{138} \mathrm{Ba} \\
(\mu \mathrm{g} / \mathrm{g})\end{array}$ & $\begin{array}{c}\mathrm{Be} \\
(\mu \mathrm{g} / \mathrm{g})\end{array}$ & $\begin{array}{c}\mathrm{Bi} \\
(\mu \mathrm{g} / \mathrm{g})\end{array}$ & $\begin{array}{c}\mathrm{Ca} \\
(\mu \mathrm{g} / \mathrm{g})\end{array}$ & $\begin{array}{l}{ }^{111} \mathrm{Cd} \\
(\mu \mathrm{g} / \mathrm{g})\end{array}$ & $\begin{array}{c}\text { Co } \\
(\mu \mathrm{g} / \mathrm{g})\end{array}$ \\
\hline B8809-17H & $<0.00903$ & 10.4 & $<0.10$ & 0.180 & $<1.04$ & NA* & 221 & $<0.017$ & $<10$ \\
\hline B8809-17H dup & $<0.0116$ & 14.1 & $<0.13$ & 0.287 & $<1.34$ & NA & 284 & $<0.022$ & $<13$ \\
\hline B8809-22C & $<0.0129$ & $<11$ & $<0.15$ & 0.272 & $<1.49$ & NA & 185 & $<0.024$ & $<15$ \\
\hline B8809-22C dup & $<0.0135$ & 21.2 & $<0.15$ & 0.285 & $<1.56$ & NA & 219 & $<0.025$ & $<16$ \\
\hline B8809-22D & $<0.0201$ & 21.1 & $<0.23$ & 0.501 & $<2.32$ & NA & 302 & $<0.037$ & $<23$ \\
\hline B8809-22D dup & $<0.104$ & 19.0 & $<0.12$ & 0.192 & $<1.20$ & NA & 146 & $<0.019$ & $<12$ \\
\hline B8809-22E & $<0.011$ & $<9.1$ & $<0.12$ & 0.257 & $<1.27$ & NA & 116 & $<0.020$ & $<13$ \\
\hline B8809-22E dup & $<0.0187$ & 21.8 & $<0.21$ & 0.513 & $<2.17$ & NA & 280 & $<0.035$ & $<22$ \\
\hline B8809-29G & $<0.0152$ & 15.2 & $<0.17$ & 0.341 & 1.75 & NA & 422 & $<0.028$ & $<18$ \\
\hline B8809-29G dup & $<0.013$ & 20.6 & $<0.15$ & 0.371 & $<1.50$ & NA & 409 & $<0.024$ & $<15$ \\
\hline B8809-29H & $<0.0104$ & 11.6 & $<0.12$ & 0.349 & $<1.20$ & NA & 320 & $<0.019$ & $<12$ \\
\hline B8809-29H dup & $<0.00975$ & 13.9 & $<0.11$ & 0.287 & $<1.13$ & NA & 321 & $<0.018$ & $<11$ \\
\hline B8809-35C & $<0.0154$ & 23.2 & $<0.17$ & 0.754 & $<1.78$ & NA & 784 & $<0.029$ & $<18$ \\
\hline B8809-35C dup & $<0.0124$ & 16.4 & $<0.14$ & 0.629 & $<1.43$ & NA & 639 & $<0.023$ & $<14$ \\
\hline B8809-35D & $<0.0143$ & 13.8 & $<0.16$ & 0.636 & $<1.65$ & NA & 828 & $<0.027$ & $<17$ \\
\hline B8809-35D dup & $<0.0131$ & 16.6 & $<0.15$ & 0.689 & $<1.51$ & NA & 708 & $<0.024$ & $<15$ \\
\hline B8809-35E & $<0.0203$ & 34.0 & $<0.23$ & 0.805 & $<2.34$ & NA & 1,020 & $<0.038$ & $<23$ \\
\hline B8809-35E dup & $<0.0178$ & 27.2 & $<0.20$ & 0.745 & $<2.06$ & NA & 1,010 & $<0.033$ & $<21$ \\
\hline B8809-39A & $<0.0113$ & 13.1 & $<0.13$ & 0.581 & $<1.31$ & NA & 404 & $<0.021$ & $<13$ \\
\hline B8809-39A dup & $<0.0103$ & 11.8 & $<0.12$ & 0.494 & $<1.19$ & NA & 366 & $<0.019$ & $<12$ \\
\hline B8809-39B & $<0.0073$ & 11.0 & $<0.082$ & 0.340 & $<0.84$ & NA & 252 & $<0.014$ & $<8.4$ \\
\hline B8809-39B dup & $<0.0126$ & 18.5 & $<0.14$ & 0.587 & $<1.45$ & NA & 378 & $<0.023$ & $<15$ \\
\hline B8809-40 & $<0.0119$ & 16.8 & $<0.13$ & 0.514 & $<1.38$ & NA & 509 & $<0.022$ & $<14$ \\
\hline B8809-40 dup & $<0.00973$ & 14.4 & $<0.11$ & 0.428 & $<1.12$ & NA & 436 & $<0.018$ & $<11$ \\
\hline B8809-40A & $<0.0144$ & 21.9 & $<0.16$ & 0.639 & $<1.66$ & NA & 400 & $<0.027$ & $<17$ \\
\hline B8809-40A dup & $<0.0123$ & 12.8 & $<0.14$ & 0.476 & $<1.42$ & NA & 321 & $<0.023$ & $<14$ \\
\hline B8809-40B & $<0.0111$ & 13.6 & $<0.12$ & 0.537 & $<1.28$ & NA & 264 & $<0.021$ & $<13$ \\
\hline B8809-40B dup & $<0.00825$ & 13.9 & $<0.093$ & 0.376 & $<0.95$ & NA & 192 & $<0.015$ & $<9.5$ \\
\hline B8809-41 & $<0.0155$ & 25.8 & $<0.17$ & 0.529 & $<1.79$ & NA & 311 & $<0.029$ & $<18$ \\
\hline B8809-41 dup & $<0.0135$ & 18.7 & $<0.15$ & 0.377 & $<1.57$ & NA & 277 & $<0.025$ & $<16$ \\
\hline B8809-42 & $<0.0129$ & 12.5 & $<0.14$ & 0.465 & $<1.49$ & NA & 264 & $<0.024$ & $<15$ \\
\hline B8809-42 dup & $<0.0119$ & 17.2 & $<0.13$ & 0.469 & $<1.38$ & NA & 224 & $<0.022$ & $<14$ \\
\hline
\end{tabular}


Table S9 (continued). SX-115 sediment sample groundwater extraction results.

\begin{tabular}{|c|c|c|c|c|c|c|c|c|c|c|}
\hline Sample ID & $\begin{array}{c}{ }^{52} \mathrm{Cr} \\
(\mu \mathrm{g} / \mathrm{g})\end{array}$ & $\begin{array}{l}{ }^{133} \mathrm{Cs} \\
(\mu \mathrm{g} / \mathrm{g})\end{array}$ & $\begin{array}{l}{ }^{65} \mathrm{Cu} \\
(\mu \mathrm{g} / \mathrm{g})\end{array}$ & $\begin{array}{c}\mathrm{Fe} \\
(\mu \mathrm{g} / \mathrm{g} \\
)\end{array}$ & $\begin{array}{c}{ }^{71} \mathrm{Ga} \\
(\mu \mathrm{g} / \mathrm{g})\end{array}$ & $\begin{array}{l}{ }^{157} \mathrm{Gd} \\
(\mu \mathrm{g} / \mathrm{g})\end{array}$ & $\begin{array}{l}{ }^{200} \mathrm{Hg} \\
(\mu \mathrm{g} / \mathrm{g})\end{array}$ & $\begin{array}{c}\mathrm{K} \\
(\mu \mathrm{g} / \mathrm{g})\end{array}$ & $\begin{array}{c}\mathrm{Mg} \\
(\mu \mathrm{g} / \mathrm{g})\end{array}$ & $\begin{array}{c}\mathrm{Mn} \\
(\mu \mathrm{g} / \mathrm{g})\end{array}$ \\
\hline B8809-17H & 0.0317 & 0.0109 & 0.0238 & $<8.4$ & $<0.012$ & $<0.012$ & $<0.039$ & $<103$ & 44.0 & $<5.0$ \\
\hline B8809-17H dup & 0.0477 & 0.0214 & 0.0437 & $<11$ & $<0.015$ & $<0.015$ & $<0.050$ & $<132$ & 64.2 & $<6.4$ \\
\hline B8809-22C & 0.721 & 0.0113 & 0.0803 & $<12$ & $<0.017$ & $<0.017$ & $<0.056$ & $<147$ & 43.9 & $<7.1$ \\
\hline B8809-22C dup & 0.668 & 0.0245 & 0.0642 & $<12$ & $<0.018$ & $<0.018$ & $<0.058$ & $<153$ & 47.6 & $<7.4$ \\
\hline B8809-22D & 3.85 & 0.0269 & 0.0642 & $<19$ & $<0.027$ & $<0.027$ & $<0.086$ & $<229$ & 69.5 & $<11$ \\
\hline B8809-22D dup & 3.27 & 0.0155 & 0.0336 & $<9.6$ & $<0.014$ & $<0.014$ & $<0.045$ & $<118$ & 25.2 & $<5.7$ \\
\hline B8809-22E & 0.157 & 0.00995 & 0.0937 & $<10$ & $<0.015$ & $<0.015$ & $<0.047$ & $<125$ & 27.1 & $<6.1$ \\
\hline B8809-22E dup & 0.164 & 0.0258 & 0.0596 & $<17$ & $<0.025$ & $<0.025$ & $<0.081$ & $<213$ & 85.2 & $<10$ \\
\hline B8809-29G & 2.32 & 0.0153 & 0.0860 & $<14$ & $<0.020$ & $<0.020$ & $<0.065$ & $<173$ & 97.1 & $<8.4$ \\
\hline B8809-29G dup & 2.57 & 0.0243 & 0.0443 & $<12$ & $<0.017$ & $<0.017$ & $<0.056$ & $<148$ & 78.2 & $<7.2$ \\
\hline B8809-29H & 2.84 & 0.0153 & 0.439 & $<9.6$ & $<0.014$ & $<0.014$ & $<0.045$ & $<118$ & 63.0 & $<5.7$ \\
\hline B8809-29H dup & 3.34 & 0.0152 & 0.0324 & $<9.0$ & $<0.013$ & $<0.013$ & $<0.042$ & $<111$ & 57.5 & $<5.4$ \\
\hline B8809-35C & 0.0636 & 0.0251 & 0.0764 & $<14$ & $<0.020$ & $<0.020$ & $<0.066$ & $<176$ & 179 & $<8.5$ \\
\hline B8809-35C dup & 0.0486 & 0.0146 & 0.0390 & 26.7 & $<0.016$ & $<0.016$ & $<0.053$ & $<141$ & 148 & $<6.8$ \\
\hline B8809-35D & 0.0469 & 0.0167 & 0.0377 & $<13$ & $<0.019$ & $<0.019$ & $<0.061$ & $<162$ & 201 & $<7.9$ \\
\hline B8809-35D dup & 0.0535 & 0.0206 & 0.0830 & $<12$ & $<0.017$ & $<0.017$ & $<0.056$ & $<149$ & 172 & $<7.2$ \\
\hline B8809-35E & 0.0792 & 0.0305 & 0.0648 & $<19$ & $<0.027$ & $<0.027$ & $<0.087$ & $<231$ & 246 & $<11$ \\
\hline B8809-35E dup & 0.0793 & 0.0289 & 0.0534 & $<17$ & $<0.024$ & $<0.024$ & $<0.077$ & $<203$ & 242 & $<9.8$ \\
\hline B8809-39A & 0.0441 & 0.0141 & 0.145 & $<11$ & $<0.015$ & $<0.015$ & $<0.049$ & $<129$ & 91.9 & $<6.3$ \\
\hline B8809-39A dup & 0.0408 & 0.00931 & 0.0915 & $<9.5$ & $<0.014$ & $<0.014$ & $<0.044$ & $<117$ & 84.9 & $<5.7$ \\
\hline B8809-39B & 0.0330 & 0.0119 & 0.0233 & $<6.8$ & $<0.0097$ & $<0.0097$ & $<0.031$ & $<83$ & 57.5 & $<4.0$ \\
\hline B8809-39B dup & 0.0580 & 0.0217 & 0.0715 & $<12$ & $<0.017$ & $<0.017$ & $<0.054$ & $<143$ & 100 & $<6.9$ \\
\hline B8809-40 & 0.0544 & 0.0197 & 0.0487 & $<11$ & $<0.016$ & $<0.016$ & $<0.051$ & $<136$ & 95.0 & $<6.6$ \\
\hline B8809-40 dup & 0.0431 & 0.0152 & 0.331 & $<9.0$ & $<0.013$ & $<0.013$ & $<0.042$ & $<111$ & 80.5 & $<5.4$ \\
\hline B8809-40A & 0.0634 & 0.0216 & 0.0649 & $<13$ & $<0.019$ & $<0.019$ & $<0.062$ & $<164$ & 113 & $<7.9$ \\
\hline B8809-40A dup & 0.0397 & 0.0121 & 0.0352 & $<11$ & $<0.016$ & $<0.016$ & $<0.053$ & $<140$ & 97.3 & $<6.8$ \\
\hline B8809-40B & 0.0390 & 0.0159 & 0.0458 & $<10$ & $<0.015$ & $<0.015$ & $<0.048$ & $<126$ & 86.8 & $<6.1$ \\
\hline B8809-40B dup & 0.0341 & 0.0145 & 0.0489 & $<7.6$ & $<0.011$ & $<0.011$ & $<0.035$ & $<94$ & 63.6 & $<4.6$ \\
\hline B8809-41 & 0.0709 & 0.0304 & 0.138 & $<14$ & $<0.021$ & $<0.021$ & $<0.066$ & $<176$ & 101 & $<8.5$ \\
\hline B8809-41 dup & 0.0458 & 0.0169 & 0.039 & $<13$ & $<0.018$ & $<0.018$ & $<0.058$ & $<154$ & 88.8 & $<7.5$ \\
\hline B8809-42 & 0.0439 & 0.0135 & 0.0391 & $<12$ & $<0.017$ & $<0.017$ & $<0.055$ & $<146$ & 76.3 & $<7.1$ \\
\hline B8809-42 dup & 0.0562 & 0.0230 & 0.0492 & $<11$ & $<0.016$ & $<0.016$ & $<0.051$ & $<136$ & 66.5 & $<6.6$ \\
\hline
\end{tabular}


Table S9 (continued). SX-115 sediment sample groundwater extraction results.

\begin{tabular}{|c|c|c|c|c|c|c|c|c|c|c|}
\hline Sample ID & $\begin{array}{l}{ }^{95} \mathrm{Mo} \\
(\mu \mathrm{g} / \mathrm{g})\end{array}$ & $\begin{array}{c}\mathrm{Na} \\
(\mu \mathrm{g} / \mathrm{g})\end{array}$ & $\begin{array}{c}{ }^{60} \mathrm{Ni} \\
(\mu \mathrm{g} / \mathrm{g})\end{array}$ & $\begin{array}{c}\mathrm{P} \\
(\mu \mathrm{g} / \mathrm{g})\end{array}$ & $\begin{array}{l}{ }^{208} \mathrm{~Pb} \\
(\mu \mathrm{g} / \mathrm{g})\end{array}$ & $\begin{array}{c}{ }^{185} \mathrm{Re} \\
(\mu \mathrm{g} / \mathrm{g})\end{array}$ & $\begin{array}{c}{ }^{101} \mathrm{Ru} \\
(\mu \mathrm{g} / \mathrm{g})\end{array}$ & $\begin{array}{c}\mathrm{S} \\
(\mu \mathrm{g} / \mathrm{g})\end{array}$ & $\begin{array}{c}{ }^{121} \mathrm{Sb} \\
(\mu \mathrm{g} / \mathrm{g})\end{array}$ & $\begin{array}{c}{ }^{82} \mathrm{Se} \\
(\mu \mathrm{g} / \mathrm{g}) \\
\end{array}$ \\
\hline B8809-17H & $<0.013$ & 331 & $<0.018$ & $<48$ & 0.00739 & $<0.0071$ & $<0.011$ & $<169$ & $<0.013$ & $<0.32$ \\
\hline B8809-17H dup & 0.0184 & 462 & $<0.023$ & $<62$ & 0.0112 & $<0.0092$ & $<0.014$ & $<217$ & $<0.016$ & $<0.42$ \\
\hline B8809-22C & 0.0359 & 675 & $<0.026$ & $<69$ & 0.0107 & $<0.010$ & $<0.016$ & $<241$ & $<0.018$ & $<0.46$ \\
\hline B8809-22C dup & 0.0413 & 932 & $<0.027$ & $<72$ & 0.0169 & $<0.011$ & $<0.017$ & $<251$ & $<0.019$ & $<0.48$ \\
\hline B8809-22D & 0.0709 & 1340 & $<0.040$ & $<107$ & 0.0189 & $<0.016$ & $<0.025$ & $<375$ & $<0.029$ & $<0.72$ \\
\hline B8809-22D dup & 0.0600 & 955 & $<0.021$ & $<55$ & 0.0107 & $<0.0082$ & $<0.013$ & $<194$ & $<0.015$ & $<0.37$ \\
\hline B8809-22E & 0.0289 & 662 & $<0.022$ & $<58$ & 0.0108 & $<0.0087$ & $<0.014$ & $<205$ & $<0.016$ & $<0.39$ \\
\hline B8809-22E dup & 0.0362 & 943 & $<0.037$ & $<100$ & 0.0166 & $<0.015$ & $<0.023$ & $<350$ & $<0.027$ & $<0.67$ \\
\hline B8809-29G & 0.0225 & 561 & $<0.030$ & $<81$ & 0.0140 & $<0.012$ & $<0.019$ & $<283$ & $<0.022$ & $<0.54$ \\
\hline B8809-29G dup & 0.0249 & 631 & $<0.026$ & $<69$ & 0.0150 & $<0.010$ & $<0.016$ & $<243$ & $<0.018$ & $<0.47$ \\
\hline B8809-29H & 0.0171 & 457 & $<0.021$ & $<55$ & 0.0104 & $<0.0082$ & $<0.013$ & $<194$ & $<0.015$ & $<0.37$ \\
\hline B8809-29H dup & 0.0177 & 508 & $<0.019$ & $<52$ & 0.0100 & $<0.0077$ & $<0.012$ & $<182$ & $<0.014$ & $<0.35$ \\
\hline B8809-35C & 0.0435 & 699 & $<0.031$ & $<82$ & 0.0200 & $<0.012$ & $<0.019$ & $<288$ & $<0.022$ & $<0.55$ \\
\hline B8809-35C dup & 0.0261 & 479 & $<0.025$ & $<66$ & 0.0113 & $<0.0098$ & $<0.015$ & $<231$ & $<0.018$ & $<0.44$ \\
\hline B8809-35D & 0.0336 & 506 & $<0.028$ & $<76$ & 0.0116 & $<0.011$ & $<0.018$ & $<267$ & $<0.020$ & $<0.51$ \\
\hline B8809-35D dup & 0.0391 & 592 & $<0.026$ & $<70$ & 0.0163 & $<0.010$ & $<0.016$ & $<244$ & $<0.019$ & $<0.47$ \\
\hline B8809-35E & 0.0517 & 909 & $<0.040$ & $<108$ & 0.0235 & $<0.016$ & $<0.025$ & $<379$ & $<0.029$ & $<0.73$ \\
\hline B8809-35E dup & 0.0473 & 828 & $<0.035$ & $<95$ & 0.0231 & $<0.014$ & $<0.022$ & $<333$ & $<0.025$ & $<0.64$ \\
\hline B8809-39A & 0.0171 & 425 & $<0.022$ & $<60$ & 0.0105 & $<0.0090$ & $<0.014$ & $<212$ & $<0.016$ & $<0.41$ \\
\hline B8809-39A dup & 0.0155 & 316 & $<0.020$ & $<55$ & 0.0116 & $<0.0082$ & $<0.013$ & $<193$ & $<0.015$ & $<0.37$ \\
\hline B8809-39B & 0.013 & 336 & $<0.014$ & $<39$ & 0.0084 & $<0.0058$ & $<0.0091$ & $<136$ & $<0.010$ & $<0.26$ \\
\hline B8809-39B dup & 0.0229 & 560 & $<0.025$ & $<67$ & 0.0139 & $<0.0099$ & $<0.016$ & $<235$ & $<0.018$ & $<0.45$ \\
\hline B8809-40 & 0.0219 & 490 & $<0.024$ & $<63$ & 0.0194 & $<0.0094$ & $<0.015$ & $<223$ & $<0.017$ & $<0.43$ \\
\hline B8809-40 dup & 0.0239 & 420 & $<0.019$ & $<52$ & 0.0118 & $<0.0077$ & $<0.012$ & $<182$ & $<0.014$ & $<0.35$ \\
\hline B8809-40A & 0.0258 & 640 & $<0.029$ & $<77$ & 0.0161 & $<0.011$ & $<0.018$ & $<269$ & $<0.020$ & $<0.52$ \\
\hline B8809-40A dup & 0.0185 & 327 & $<0.024$ & $<65$ & 0.0103 & $<0.0097$ & $<0.015$ & $<230$ & $<0.017$ & $<0.44$ \\
\hline B8809-40B & 0.0176 & 423 & $<0.022$ & $<59$ & 0.00908 & $<0.0088$ & $<0.014$ & $<208$ & $<0.016$ & $<0.40$ \\
\hline B8809-40B dup & 0.0151 & 327 & $<0.016$ & $<44$ & 0.00885 & $<0.0065$ & $<0.010$ & $<154$ & $<0.012$ & $<0.30$ \\
\hline B8809-41 & 0.0282 & 692 & $<0.031$ & $<82$ & 0.0198 & $<0.012$ & $<0.019$ & $<289$ & $<0.022$ & $<0.55$ \\
\hline B8809-41 dup & 0.0204 & 450 & $<0.027$ & $<72$ & 0.0105 & $<0.011$ & $<0.017$ & $<253$ & $<0.019$ & $<0.49$ \\
\hline B8809-42 & $<0.018$ & 418 & $<0.025$ & $<68$ & 0.00927 & $<0.010$ & $<0.016$ & $<240$ & $<0.018$ & $<0.46$ \\
\hline B8809-42 dup & 0.0263 & 534 & $<0.024$ & $<63$ & 0.0150 & $<0.0094$ & $<0.015$ & $<223$ & $<0.017$ & $<0.43$ \\
\hline
\end{tabular}


Table S9 (continued). SX-115 sediment sample groundwater extraction results.

\begin{tabular}{|c|c|c|c|c|c|c|c|c|}
\hline Sample ID & $\begin{array}{c}\mathrm{Si} \\
(\mu \mathrm{g} / \mathrm{g})\end{array}$ & $\begin{array}{c}\mathrm{Sr} \\
(\mu \mathrm{g} / \mathrm{g})\end{array}$ & $\begin{array}{l}{ }^{232} \mathrm{Th} \\
(\mu \mathrm{g} / \mathrm{g})\end{array}$ & $\begin{array}{c}\mathrm{Ti} \\
(\mu \mathrm{g} / \mathrm{g})\end{array}$ & $\begin{array}{c}{ }^{238} \mathrm{U} \\
(\mu \mathrm{g} / \mathrm{g})\end{array}$ & $\begin{array}{c}\mathrm{V} \\
(\mu \mathrm{g} / \mathrm{g})\end{array}$ & $\begin{array}{c}\mathrm{Zn} \\
(\mu \mathrm{g} / \mathrm{g})\end{array}$ & $\begin{array}{c}\mathrm{Zr} \\
(\mu \mathrm{g} / \mathrm{g}) \\
\end{array}$ \\
\hline B8809-17H & NR & $<6.4$ & $<0.0017$ & $<7.9$ & 0.00701 & $<11$ & $<7.8$ & 10 \\
\hline B8809-17H dup & NR & $<8.2$ & $<0.0022$ & $<10$ & 0.00739 & $<12$ & $<10$ & 13 \\
\hline B8809-22C & NR & $<9.1$ & $<0.0025$ & $<11$ & 0.0101 & $<15$ & $<11$ & 15 \\
\hline B8809-22C dup & NR & $<9.5$ & $<0.0026$ & $<12$ & 0.00961 & $<16$ & $<12$ & 15 \\
\hline B8809-22D & NR & $<14$ & $<0.0039$ & $<18$ & 0.0165 & $<24$ & $<17$ & 23 \\
\hline B8809-22D dup & NR & $<7.3$ & 0.00350 & $<9.1$ & 0.00882 & $<12$ & $<9.0$ & 12 \\
\hline B8809-22E & NR & $<7.8$ & $<0.0021$ & $<9.6$ & 0.0106 & $<13$ & $<9.5$ & 13 \\
\hline B8809-22E dup & NR & $<13$ & $<0.0036$ & $<17$ & 0.0158 & $<22$ & $<16$ & 21 \\
\hline B8809-29G & NR & $<11$ & $<0.0029$ & $<13$ & 0.0118 & $<18$ & $<13$ & 17 \\
\hline B8809-29G dup & NR & $<9.2$ & $<0.0025$ & $<11$ & 0.00998 & $<15$ & $<11$ & 15 \\
\hline В8809-29H & NR & $<7.3$ & 0.00228 & $<9.1$ & 0.00869 & $<12$ & $<8.9$ & 12 \\
\hline B8809-29H dup & NR & $<6.9$ & 0.00200 & $<8.6$ & 0.00824 & $<12$ & $<8.4$ & 11 \\
\hline B8809-35C & NR & $<11$ & $<0.0030$ & $<14$ & 0.0115 & $<18$ & $<13$ & 18 \\
\hline B8809-35C dup & NR & $<8.7$ & $<0.0024$ & $<11$ & 0.0088 & $<15$ & $<11$ & 14 \\
\hline B8809-35D & NR & $<10$ & 0.00370 & $<13$ & 0.291 & $<17$ & $<12$ & 16 \\
\hline B8809-35D dup & NR & $<9.2$ & $<0.0025$ & $<12$ & 0.00985 & $<15$ & $<11$ & 15 \\
\hline B8809-35E & NR & $<14$ & 0.00660 & $<18$ & 0.0167 & $<24$ & $<18$ & 23 \\
\hline B8809-35E dup & NR & $<13$ & 0.00488 & $<16$ & 0.0143 & $<21$ & $<15$ & 20 \\
\hline B8809-39A & NR & $<8.0$ & $<0.0022$ & $<10$ & 0.0212 & $<13$ & $<9.8$ & 13 \\
\hline B8809-39A dup & NR & $<7.3$ & $<0.0020$ & $<9.0$ & 0.0195 & $<12$ & $<8.9$ & 12 \\
\hline B8809-39B & NR & $<5.2$ & $<0.0014$ & $<6.4$ & 0.012 & $<8.6$ & $<6.3$ & 8.3 \\
\hline B8809-39B dup & NR & $<8.9$ & $<0.0024$ & $<11$ & 0.0162 & $<15$ & $<11$ & 14 \\
\hline B8809-40 & NR & $<8.4$ & $<0.0023$ & $<11$ & 0.0867 & $<14$ & $<10$ & 14 \\
\hline B8809-40 dup & NR & $<6.9$ & $<0.0019$ & $<8.5$ & 0.0851 & $<11$ & $<8.4$ & 11 \\
\hline B8809-40A & NR & $<10$ & $<0.0028$ & $<13$ & 0.0245 & $<17$ & $<12$ & 16 \\
\hline B8809-40A dup & NR & $<8.7$ & $<0.0024$ & $<11$ & 0.0223 & $<14$ & $<11$ & 14 \\
\hline B8809-40B & NR & $<7.9$ & $<0.0021$ & $<9.7$ & 0.0162 & $<13$ & $<9.6$ & 13 \\
\hline B8809-40B dup & NR & $<5.8$ & $<0.0016$ & $<7.2$ & 0.0117 & $<9.7$ & $<7.1$ & 9.4 \\
\hline B8809-41 & NR & $<11$ & $<0.0030$ & $<14$ & 0.00768 & $<18$ & $<13$ & 18 \\
\hline B8809-41 dup & NR & $<9.6$ & $<0.0026$ & $<12$ & 0.00786 & $<16$ & $<12$ & 15 \\
\hline B8809-42 & NR & $<9.1$ & $<0.0025$ & $<11$ & 0.0189 & $<15$ & $<11$ & 15 \\
\hline B8809-42 dup & NR & $<8.4$ & $<0.0023$ & $<11$ & 0.0116 & $<14$ & $<10$ & 14 \\
\hline
\end{tabular}


Table S10. Major elements in acid extracts ( $\mu \mathrm{g} / \mathrm{g}$ ) for composite, grab sediment samples as measured previously [13]

\begin{tabular}{ccccc}
\hline Element & $\begin{array}{c}\text { Ringold } \\
\text { Silt }\end{array}$ & $\begin{array}{c}\text { Hanford } \\
\text { Coarse Sand }\end{array}$ & $\begin{array}{c}\text { Hanford } \\
\text { Fine Sand }\end{array}$ & $\begin{array}{c}\text { Borehole } \\
\text { Fine Sand }\end{array}$ \\
\hline $\mathrm{Na}$ & 191 & 211 & 280 & 225 \\
$\mathrm{Mg}$ & 12553 & 3029 & 6537 & 5690 \\
$\mathrm{Al}$ & 23256 & 4067 & 11908 & 8954 \\
$\mathrm{Si}$ & 11.1 & 82.3 & 20 & 38.1 \\
$\mathrm{P} *$ & 316 & 766 & 647 & 539 \\
$\mathrm{~S}$ & 43.5 & 62.7 & 142.5 & 47.9 \\
$\mathrm{I}$ & 2455 & 704 & 2079 & 1915 \\
$\mathrm{Ca}$ & 15168 & 4922 & 11101 & 9674 \\
$\mathrm{Ti}$ & 541 & 681 & 1029 & 696 \\
$\mathrm{Mn}$ & 648 & 187 & 429 & 316 \\
$\mathrm{Fe}$ & 34626 & 21814 & 19741 & 15094 \\
$\mathrm{Sr}$ & 41.2 & 19.9 & 50.8 & 37.3 \\
$\mathrm{Ba}$ & 110 & 43.9 & 97.3 & 81.2 \\
\hline
\end{tabular}

${ }^{*} \mathrm{P}$ data is suspect due to spectra interferences

Table S11. Trace metals in acid extracts $(\mu \mathrm{g} / \mathrm{g})$ for composite, grab sediment samples as measured previously [13]

\begin{tabular}{ccccc}
\hline Element & $\begin{array}{c}\text { Ringold } \\
\text { Silt }\end{array}$ & $\begin{array}{c}\text { Hanford } \\
\text { Coarse Sand }\end{array}$ & $\begin{array}{c}\text { Hanford } \\
\text { Fine Sand }\end{array}$ & $\begin{array}{c}\text { Borehole } \\
\text { Fine Sand }\end{array}$ \\
\hline $\mathrm{Be}$ & 0.94 & 0.16 & 0.41 & 0.34 \\
$\mathrm{~B}$ & 2.09 & 10.42 & 7.25 & 8.55 \\
$\mathrm{Cr}$ & 39.7 & 4.3 & 17.2 & 14.9 \\
$\mathrm{Co}$ & 16.2 & 6.5 & 11.5 & 7.6 \\
$\mathrm{Ni}$ & 35.4 & 5.7 & 16.6 & 12.8 \\
$\mathrm{Cu}$ & 27.4 & 9.2 & 12.7 & 8.9 \\
$\mathrm{As}$ & 15.9 & 1 & 2.9 & 3.9 \\
$\mathrm{Se}$ & $<0.3$ & $<0.3$ & $<0.3$ & $<0.3$ \\
$\mathrm{Mo}$ & 0.27 & 0.14 & 0.17 & 0.24 \\
$\mathrm{Ru}$ & $<0.001$ & $<0.001$ & $<0.001$ & $<0.001$ \\
$\mathrm{Ag}$ & 0.022 & 0.038 & 0.055 & 0.045 \\
$\mathrm{Cd}$ & 0.137 & 0.044 & 0.077 & 0.08 \\
$\mathrm{~Pb}$ & 14.9 & 2.45 & 6.38 & 5.93 \\
$\mathrm{U}$ & 0.95 & 0.32 & 0.59 & 0.62 \\
\hline
\end{tabular}


Table S12. Major elements in acid extracts $(\mu \mathrm{g} / \mathrm{g})$ for borehole 299-W22-48 as measured previously [13], where $\mathrm{H}-$ Hanford formation, PPI - Pleistocene formation, and Rtf Ringold formation

\begin{tabular}{|c|c|c|c|c|c|c|c|c|c|c|}
\hline $\begin{array}{l}\text { Depth (ft) } \\
\text { Formation }\end{array}$ & $\begin{array}{r}39.5 \\
\text { H1a } \\
\end{array}$ & $\begin{array}{r}47 \\
\text { H1 } \\
\end{array}$ & $\begin{array}{r}77 \\
\text { H2 } \\
\end{array}$ & $\begin{array}{l}91.5 \\
\mathrm{H} 2 \\
\end{array}$ & $\begin{array}{c}101.5 \\
\mathrm{H} 2 \\
\end{array}$ & $\begin{array}{l}136 \\
\text { PPlz }\end{array}$ & $\begin{array}{l}146 \\
\text { PPlc }\end{array}$ & $\begin{array}{l}148.5 \\
\text { PPIc }\end{array}$ & $\begin{array}{c}163.5 \\
\text { Rtf }\end{array}$ & $\begin{array}{l}192 \\
\text { Rtf }\end{array}$ \\
\hline $\mathrm{Na}$ & 180.5 & 278 & 204.2 & 150.2 & $\begin{array}{l}197.9 \\
\end{array}$ & 185.1 & 363.6 & 355.8 & 168.6 & 295 \\
\hline $\mathrm{Mg}$ & 4570 & 2245 & 5133 & 4022 & 5214 & 6551 & 20148 & 9586 & 4770 & 3700 \\
\hline $\mathrm{Al}$ & 6281 & 3264 & 7623 & 5394 & 7257 & 9935 & 6939 & 5950 & 6844 & 6023 \\
\hline $\mathrm{Si}$ & 35.8 & 20.1 & 26.8 & 85.5 & 14.7 & 7.5 & 11 & 17.8 & 15.8 & 16.4 \\
\hline $\mathrm{P}$ & 389.1 & 535.4 & 467.9 & 362.8 & 416.2 & 450.3 & 423.8 & 539 & 319.9 & 446.7 \\
\hline S & 45 & 39.6 & 31.5 & 31.4 & 42 & 27.5 & 353.4 & 373.2 & 18.7 & 36.3 \\
\hline K & 1488 & 446.6 & 1467 & 1214 & 1522 & 1912 & 912.9 & 810 & 857.2 & 969.4 \\
\hline $\mathrm{Ca}$ & 7611 & 5209 & 8141 & 6680 & 8410 & 11447 & 148662 & 203457 & 4439 & 2808 \\
\hline $\mathrm{Ti}$ & 541.3 & 563.1 & 527.7 & 330 & 512.8 & 446.8 & 497.3 & 380.4 & 537.1 & 622.6 \\
\hline $\mathrm{Mn}$ & 275.5 & 122.2 & 273.3 & 234.5 & 274.3 & 274.5 & 1459.1 & 230.2 & 257.8 & 193.8 \\
\hline $\mathrm{Fe}$ & 12200 & 9397 & 12885 & 10049 & 12490 & 14524 & 9805 & 8776 & 13089 & 11767 \\
\hline $\mathrm{Sr}$ & 29.1 & 18.3 & 35.2 & 24.6 & 33.9 & 36.7 & 295.2 & 390.6 & 21.2 & 22 \\
\hline $\mathrm{Ba}$ & 80.3 & 43.8 & 69.3 & 60.5 & 80.4 & 107.2 & 128 & 73.2 & 46.9 & 113.3 \\
\hline
\end{tabular}

Table S13. Trace elements in acid extracts $(\mu \mathrm{g} / \mathrm{g})$ for borehole $299-\mathrm{W} 22-48$ as measured previously [13], where $\mathrm{H}$ - Hanford formation, PPI - Pleistocene formation, and Rtf Ringold formation

\begin{tabular}{|c|c|c|c|c|c|c|c|c|c|c|}
\hline $\begin{array}{l}\text { Depth (ft) } \\
\text { Formation }\end{array}$ & $\begin{array}{l}39.5 \\
\text { H1a } \\
\end{array}$ & $\begin{array}{l}47 \\
\text { H1 }\end{array}$ & $\begin{array}{r}77 \\
\text { H2 } \\
\end{array}$ & $\begin{array}{l}91.5 \\
\mathrm{H} 2 \\
\end{array}$ & $\begin{array}{c}101.5 \\
\mathrm{H} 2\end{array}$ & $\begin{array}{l}136 \\
\text { PPlz }\end{array}$ & $\begin{array}{l}146 \\
\text { PPIc }\end{array}$ & $\begin{array}{l}148.5 \\
\text { PPIc }\end{array}$ & $\begin{array}{c}163.5 \\
\text { Rtf }\end{array}$ & $\begin{array}{l}192 \\
\text { Rtf }\end{array}$ \\
\hline $\mathrm{Be}$ & 0.23 & 0.14 & 0.27 & 0.21 & 0.24 & 0.39 & 0.22 & 0.19 & 0.24 & 0.21 \\
\hline B & 8.66 & 1.76 & 9.35 & 7.02 & 7.36 & 1.11 & 10.32 & 15.13 & 8.62 & 1.79 \\
\hline $\mathrm{Cr}$ & 8.47 & 3.21 & 13.86 & 9.49 & 12.68 & 14.17 & 10.84 & 11.77 & 8.63 & 9.81 \\
\hline Co & 5.82 & 4.56 & 6.61 & 5.09 & 6.05 & 8.23 & 5.51 & 4.68 & 5.64 & 6 \\
\hline $\mathrm{Ni}$ & 7.29 & 4.25 & 13.07 & 9.19 & 11.19 & 13.26 & 9.81 & 9.25 & 8.04 & 8.64 \\
\hline $\mathrm{Cu}$ & 7.26 & 7.61 & 8.93 & 8.34 & 7.92 & 13.5 & 16.93 & 9.9 & 6.59 & 9.21 \\
\hline $\mathrm{Zn}$ & 33.34 & 17.94 & 36.72 & 29.1 & 33.83 & 45.15 & 24.58 & 24.79 & 32.98 & 26.44 \\
\hline As & 2.25 & 0.69 & 4.41 & 3.02 & 2.99 & 4.5 & 5.96 & 19.07 & 2.39 & 1.2 \\
\hline $\mathrm{Se}$ & 0.16 & 0.22 & 0.16 & 0.15 & 0.14 & 0.22 & 0.54 & 0.71 & 0.14 & 0.17 \\
\hline Mo & 0.05 & 0.05 & 0.09 & 0.07 & 0.08 & 0.06 & 0.33 & 0.08 & 0.07 & 0.78 \\
\hline
\end{tabular}




\begin{tabular}{ccccccccccc}
$\mathrm{Ru}$ & 0.002 & 0.002 & 0.002 & 0.002 & 0.001 & 0.001 & 0.004 & 0.005 & 0.002 & 0.001 \\
$\mathrm{Ag}$ & 0.03 & 0.03 & 0.04 & 0.04 & 0.04 & 0.06 & 0.06 & 0.05 & 0.02 & 0.04 \\
$\mathrm{Cd}$ & 0.07 & 0.04 & 0.06 & 0.07 & 0.06 & 0.12 & 0.09 & 0.16 & 0.05 & 0.04 \\
$\mathrm{~Pb}$ & 4.42 & 1.57 & 5.47 & 4.58 & 4.66 & 9.04 & 3.19 & 3.24 & 4.74 & 3.99 \\
$\mathrm{U}$ & 0.53 & 0.25 & 0.59 & 0.5 & 0.48 & 0.68 & 3.55 & 3.22 & 0.53 & 0.48 \\
\hline
\end{tabular}

*Element concentrations in italics were below detection limits but were reported due to sufficient peaks above background in ICP results 
Table S14. Major elements in acid extracts $(\mu \mathrm{g} / \mathrm{g})$ for borehole 299-W22-50 as measured previously [13], where $\mathrm{H}-\mathrm{Hanford}$ formation, PPI - Pleistocene formation, and Rtf - Ringold formation

\begin{tabular}{|c|c|c|c|c|c|c|c|c|c|c|c|c|}
\hline $\begin{array}{c}\text { Depth (ft) } \\
\text { Formation }\end{array}$ & $\begin{array}{l}\text { H1a } \\
27.5 \\
\end{array}$ & $\begin{array}{c}\text { H1a } \\
51\end{array}$ & $\begin{array}{c}\text { H1 } \\
54.5 \\
\end{array}$ & $\begin{array}{l}\text { H1 } \\
60 \\
\end{array}$ & $\begin{array}{c}\text { H2 } \\
67.5 \\
\end{array}$ & $\begin{array}{c}\mathrm{H} 2 \\
96 \\
\end{array}$ & $\begin{array}{l}\mathrm{H} 2 \\
116\end{array}$ & $\begin{array}{c}\mathrm{H} 2 \\
135 \\
\end{array}$ & $\begin{array}{c}\text { PPIc } \\
140 \\
\end{array}$ & $\begin{array}{c}\text { PPIc } \\
141 \\
\end{array}$ & $\begin{array}{c}\text { Rtf } \\
150.5\end{array}$ & $\begin{array}{r}\text { Rtf } \\
160.5 \\
\end{array}$ \\
\hline $\mathrm{Na}$ & 332.4 & 425.2 & 240.2 & 309.4 & 209.4 & 218.7 & 227.8 & 272 & 212.6 & 252.9 & 365.3 & 251.7 \\
\hline $\mathrm{Mg}$ & 5523 & 5318 & 4293 & 2717 & 6360 & 5137 & 6507 & 7766 & 7019 & 5020 & 3333 & 3823 \\
\hline $\mathrm{Al}$ & 8245 & 10251 & 6311 & 3711 & 9097 & 8388 & 10940 & 16268 & 9828 & 6220 & 5402 & 5939 \\
\hline $\mathrm{Si}$ & 64.67 & 18.22 & 37.68 & 10.56 & 23.37 & 27.19 & 4.59 & 13.34 & 13.46 & 11 & 22.83 & 32.66 \\
\hline $\mathrm{P}$ & 915.7 & 773 & 630.8 & 834.4 & 565.2 & 502.5 & 553 & 484.9 & 864.6 & 489.8 & 507.1 & 510.9 \\
\hline $\mathrm{S}$ & 73.75 & 71.86 & 49.1 & 229.95 & 51.92 & 33.36 & 25.78 & 27 & 32.27 & 102.13 & 36.99 & 37.47 \\
\hline $\mathrm{K}$ & 1486 & 1435 & 1132 & 561 & 1998 & 1696 & 2302 & 2541 & 1926 & 887 & 718 & 701 \\
\hline $\mathrm{Ca}$ & 10577 & 11316 & 8421 & 14424 & 10802 & 8622 & 9991 & 13341 & 11254 & 34412 & 3862 & 3954 \\
\hline $\mathrm{Ti}$ & 1203.2 & 1818.8 & 789.1 & 464.9 & 601.1 & 677.1 & 748.1 & 872.1 & 507.9 & 470.7 & 809 & 783.6 \\
\hline $\mathrm{Mn}$ & 424.4 & 444.5 & 293.7 & 144.9 & 349.3 & 280.4 & 310.1 & 412.3 & 300.3 & 430 & 198.7 & 226.9 \\
\hline $\mathrm{Fe}$ & 23264 & 26451 & 14965 & 10619 & 15069 & 12898 & 17210 & 21168 & 16361 & 10439 & 11380 & 12401 \\
\hline $\mathrm{Sr}$ & 42.32 & 47.63 & 33.07 & 31.44 & 43.61 & 37.12 & 46.75 & 45.17 & 38.45 & 83.65 & 25.88 & 24.99 \\
\hline $\mathrm{Ba}$ & 121.5 & 107.1 & 74.7 & 63.2 & 80.3 & 74.4 & 101.9 & 124.5 & 109.1 & 78.4 & 48.8 & 50.5 \\
\hline
\end{tabular}

Table S15. Trace metals in acid extracts $(\mu \mathrm{g} / \mathrm{g})$ for borehole 299-W22-50 as measured previously [13], where $\mathrm{H}-\mathrm{Hanford}$ formation, PPI - Pleistocene formation, and Rtf - Ringold formation

\begin{tabular}{|c|c|c|c|c|c|c|c|c|c|c|c|c|}
\hline $\begin{array}{l}\text { Depth (ft) } \\
\text { Formation } \\
\end{array}$ & $\begin{array}{l}\text { H1a } \\
27.5\end{array}$ & $\begin{array}{c}\text { H1a } \\
51 \\
\end{array}$ & $\begin{array}{c}\text { H1 } \\
54.5\end{array}$ & $\begin{array}{l}\text { H1 } \\
60 \\
\end{array}$ & $\begin{array}{c}\mathrm{H} 2 \\
67.5 \\
\end{array}$ & $\begin{array}{l}\mathrm{H2} \\
96 \\
\end{array}$ & $\begin{array}{l}\mathrm{H} 2 \\
116 \\
\end{array}$ & $\begin{array}{c}\mathrm{H} 2 \\
135 \\
\end{array}$ & $\begin{array}{c}\text { PPIc } \\
140 \\
\end{array}$ & $\begin{array}{c}\text { PPIc } \\
141 \\
\end{array}$ & $\begin{array}{c}\text { Rtf } \\
150.5\end{array}$ & $\begin{array}{c}\text { Rtf } \\
160.5\end{array}$ \\
\hline $\mathrm{Be}$ & 0.37 & 0.33 & 0.4 & 0.24 & 0.16 & 0.33 & 0.3 & 0.36 & 0.56 & 0.4 & 0.22 & 0.17 \\
\hline B & 1.64 & 0.28 & 3.86 & 4.98 & 9.7 & 6.38 & 2.72 & 5.55 & 4.75 & 7.56 & 3.78 & 4.98 \\
\hline $\mathrm{Cr}$ & 9.78 & 8.97 & 8.78 & 4.25 & 14.96 & 15.69 & 19.49 & 19.32 & 16.65 & 9.36 & 7.44 & 7.88 \\
\hline Co & 12.17 & 10.91 & 13.79 & 7.74 & 5.62 & 7.65 & 6.86 & 8.7 & 10.48 & 8.99 & 7.15 & 6.41 \\
\hline $\mathrm{Ni}$ & 11.17 & 10.33 & 8.94 & 8.49 & 5.36 & 13.69 & 12.61 & 17.45 & 15.87 & 16.94 & 9.7 & 8.25 \\
\hline $\mathrm{Cu}$ & 10.91 & 10.86 & 9.45 & 7.92 & 10.58 & 9.98 & 8.41 & 13.38 & 18.79 & 17.23 & 10.86 & 9.17 \\
\hline $\mathrm{Zn}$ & 47.71 & 43.65 & 47.5 & 32.14 & 35.96 & 44.08 & 35.76 & 49.19 & 55.24 & 45.14 & 25.27 & 25.39 \\
\hline As & 4.76 & 2.04 & 1.65 & 1 & 3.78 & 4.02 & 6.16 & 4.56 & 2.65 & 3.64 & 1.06 & 1.3 \\
\hline $\mathrm{Se}$ & 0.35 & 0.33 & 0.24 & 0.35 & 0.15 & 0.16 & 0.18 & 0.27 & 0.29 & 0.16 & 0.22 & 0.17 \\
\hline Mo & 0.1 & 0.09 & 0.09 & 0.12 & 0.1 & 0.09 & 0.1 & 0.09 & 0.08 & 0.28 & 0.22 & 0.14 \\
\hline $\mathrm{Ru}$ & 0.001 & 0.001 & 0.001 & 0.001 & 0.001 & 0.001 & 0.001 & 0.001 & 0.002 & 0.001 & 0.001 & 0.001 \\
\hline $\mathrm{Ag}$ & 0.06 & 0.06 & 0.05 & 0.05 & 0.05 & 0.04 & 0.05 & 0.06 & 0.07 & 0.03 & 0.03 & 0.05 \\
\hline
\end{tabular}




\begin{tabular}{ccccccccccccc}
$\mathrm{Cd}$ & 0.09 & 0.08 & 0.06 & 0.06 & 0.07 & 0.06 & 0.12 & 0.14 & 0.11 & 0.07 & 0.04 & 0.05 \\
$\mathrm{~Pb}$ & 5.06 & 4.28 & 3.69 & 2.65 & 6.28 & 5.16 & 6.31 & 10.45 & 8.34 & 2.93 & 2.52 & 3.96 \\
$\mathrm{U}$ & 0.82 & 0.68 & 0.51 & 0.45 & 0.74 & 0.82 & 0.78 & 0.97 & 1.2 & 1.29 & 0.4 & 0.39 \\
\hline
\end{tabular}

*Element concentrations in italics were below detection limits but were reported due to sufficient peaks above background in ICP results 


\section{References}

1. Truex, M.J., et al., Conceptual Model of lodine Behavior in the Subsurface at the Hanford Site. 2017, Pacific Northwest National Lab: Richland, WA.

2. $\quad$ EPA, Inductively Coupled Plasma Mass Spectrometry, in Test Method 6020A. 2007, Environmental Protection Agency: Washington, D.C.

3. Laboratory, P.N.N., Inductively Coupled Plasma Mass Spectrometry, in Technical Procedure. 2016, Pacific Northwest National Laboratory: Richland, WA.

4. Laboratory, P.N.N., Inductively Coupled Plasma Optical Emission Spectrometry, in Technical Procedure. 2016, Pacific Northwest National Laboratory: Richland, WA.

5. EPA, Inductively Coupled Plasma Atomic Emission Spectrometry, in Test Method 6010C. 2007, Environmental Protection Agency: Washington, D.C.

6. Association, A.P.H. and A.W.W. Association, Standard methods for the examination of water and wastewater. 1989: American public health association.

7. Laboratory, P.N.N., Alkalinity Measurements, in Technical Procedure. 2016, Pacific Northwest National Laboratory: Richland, WA.

8. Laboratory, P.N.N., Ion Chromatography Analysis, in Technical Procedure. 2014, Pacific Northwest National Laboratory: Richland, WA.

9. EPA, Determination of Inorganic Anions by Ion Chromatography, in Test Method 300.0. 1993, Environmental Protection Agency: Washington, D.C.

10. Delegard, C., et al., Generation and characteristics of plutonium and americium contaminated soils underlying waste sites at Hanford 2019, Pacific Northwest National Laboratory: Richland, WA.

11. Delegard, C.H., S.A. Gallagher, and R.B. Kasper. Saturated Column Leach Studies: Hanford 216Z-1A Sedimet. in International Symposium on the Migration in the Terrestrial Environment of Long-Lived Radionuclides. 1981. International Atomic Energy Agency.

12. Rai, D., et al., Influence of an Americium Solid Phase on Americium Concentrations in Solutions. Geochimica et Cosmochimica Acta, 1981. 45: p. 2257-2265.

13. Serne, R.J., et al., Characterization of Vadose Zone Sediment: Borehole 299-W23-19 [SX-115] in the S-SX Waste Management Area. 2002, Pacific Northwest National Lab.(PNNL), Richland, WA (United States).

14. Serne, R.J., et al., Characterization of vadose zone sediment: Borehole 299-E33-45 near BX-102 in the $B-B X-B Y$ waste management area. 2008, Pacific Northwest National Laboratory, Richland, WA.

15. Felmy, A.R., K.J. Cantrell, and S.D. Conradson, Plutonium contamination issues in Hanford soils and sediments: Discharges from the Z-Plant (PFP) comples. Phys Chem Earth, 2010. 35(6-8): p. 292-297. 